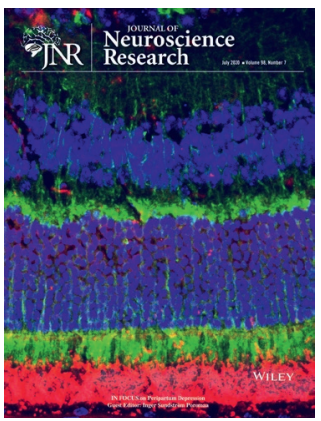

\title{
GDF15 promotes simultaneous astrocyte remodeling and tight junction strengthening at the blood-brain barrier
}

\author{
Victoria A. Malik $^{1}$ | Franziska Zajicek ${ }^{1}$ | Laura A. Mittmann ${ }^{1}$ | Johannes Klaus ${ }^{2}$ | \\ Sandra Unterseer ${ }^{2}$ | Sandeep Rajkumar ${ }^{1}$ | Benno Pütz ${ }^{2}$ | Jan M. Deussing ${ }^{2}$ | \\ Inga D. Neumann ${ }^{3,4}$ | Rainer Rupprecht ${ }^{1,4}$ | Barbara Di Benedetto ${ }^{1,4}$ (ID
}

${ }^{1}$ Department of Psychiatry and Psychotherapy, University of Regensburg, Regensburg, Germany

${ }^{2}$ Max Planck Institute of Psychiatry, Munich, Germany

${ }^{3}$ Department of Neurobiology and Animal Physiology, University of Regensburg, Regensburg, Germany

${ }^{4}$ Regensburg Center of Neuroscience, University of Regensburg, Regensburg, Germany

\section{Correspondence}

Barbara Di Benedetto, Department of Psychiatry and Psychotherapy, University of Regensburg, Franz-Josef-Strauß Allee 11, 93053 Regensburg, Germany.

Email: Barbara.Di-Benedetto@ukr.de

Funding information

Deutsche Forschungsgemeinschaft, Grant/Award Number: GRK2174; Bundesministerium für Bildung und Forschung, Grant/Award Number: 01EE1401A and 01EE1401B; Universität Regensburg, Grant/Award Number: Intramural funding

\begin{abstract}
Perivascular astrocyte processes (PAP) surround cerebral endothelial cells (ECs) and modulate the strengthening of tight junctions to influence blood-brain barrier (BBB) permeability. Morphologically altered astrocytes may affect barrier properties and trigger the onset of brain pathologies. However, astrocyte-dependent mediators of these events remain poorly studied. Here, we show a pharmacologically driven elevated expression and release of growth/differentiation factor 15 (GDF15) in rat primary astrocytes and cerebral PAP. GDF15 has been shown to possess trophic properties for motor neurons, prompting us to hypothesize similar effects on astrocytes. Indeed, its increased expression and release occurred simultaneously to morphological changes of astrocytes in vitro and PAP, suggesting modulatory effects of GDF15 on these cells, but also neighboring EC. Administration of recombinant GDF15 was sufficient to promote astrocyte remodeling and enhance barrier properties between ECs in vitro, whereas its pharmacogenetic abrogation prevented these effects. We validated our findings in male high anxiety-related behavior rats, an animal model of depressive-like behavior, with shrunk PAP associated with reduced expression of the junctional protein claudin-5, which were both restored by a pharmacologically induced increase in GDF15 expression. Thus, we identified GDF15 as an astrocytederived trigger of astrocyte process remodeling linked to enhanced tight junction strengthening at the BBB.
\end{abstract}

\section{KEYWORDS}

astrocytes, blood-brain barrier, GDF15, remodeling, RRID:SCR_003070, RRID:AB_10753223, RRID:RGD_13508588, RRID:AB_2242334, RRID:AB_2534069, RRID:AB_258785,

RRID:AB_297817, RRID:AB_477010, RRID:AB_732535, tight junctions

Edited by Jerome Badaut. Statistics Editor: David McArthur. Reviewed by Richard Keep. 


\section{1 | INTRODUCTION}

Astrocytes regulate various physiological processes, including synaptic communication and functionality of the blood-brain barrier (BBB), through either released factors or contact-dependent effectors located on their processes (Boulay et al., 2017; Cabezas et al., 2014; Obermeier, Daneman, \& Ransohoff, 2013; Stogsdill et al., 2017). The tightly regulated interaction between the endfeet of astrocyte processes and endothelial cells (ECs) around blood vessels (BVs) is crucial to enhance the formation of multiprotein complexes known as tight junctions (TJ), which are in turn essential for the strengthening of a fully functional BBB. Although it is still a matter of debate whether astrocytes are indispensable for the initial steps of TJ development (Saunders, Dziegielewska, Unsicker, \& Ek, 2016), it is generally accepted that they are required to reinforce barrier properties, as demonstrated by the higher vascular permeability of meningeal BVs devoid of direct contacts with glia cells (Alvarez et al., 2011). Besides astrocytes, pericytes also are involved in the regulation of both development and maintenance of the BBB. Several studies have evidenced the importance of pericytes for multiple functions, including the support of BBB integrity, angiogenesis, clearance of toxic metabolites, and control of hemodynamic responses of BVs (Mazare, Gilbert, Boulay, Rouach, \& Cohen-Salmon, 2018). TJ connect ECs and influence paracellular fence and gate functions of the BBB, thus monitoring the transport of molecules between blood and brain parenchyma (Alvarez, Katayama, \& Prat, 2013; Omidi \& Barar, 2012). Any disruption in the structural integrity of astrocytic endfeet or junctional protein assemblies can lead to perturbed barrier properties and the onset of severe brain pathologies (Alvarez et al., 2011; Menard et al., 2017; Obermeier et al., 2013; Reuss, Dono, \& Unsicker, 2003; Watkins et al., 2014). Indeed, an altered astrocyte morphology characterize several neurological and neuropsychiatric disorders (Di Benedetto \& Rupprecht, 2013; Lee \& MacLean, 2015; Pekny et al., 2016; Watkins et al., 2014). Recent work revealed a disrupted glia cell morphology, marked by reduced expression of aquaporin- 4 in the endfeet of perivascular astrocyte processes (PAP), in the prefrontal cortex (PFC) of major depressive disorder (MDD) patients (Rajkowska, Hughes, Stockmeier, Javier Miguel-Hidalgo, \& Maciag, 2013). We confirmed those findings in rats selectively bred for high anxiety-related behavior (HAB), a model of innate depression (Wegener, Mathe, \& Neumann, 2012), which additionally showed a remarkable shrinkage of entire astrocyte processes (Barbara Di Benedetto et al., 2016). Our observations suggested that the delayed clinical efficacy of pharmacotherapies might depend on a perturbed permeability of the BBB to treatment drugs possibly caused by an altered astrocyte morphology around BVs. The antidepressant (AD) fluoxetine (FLX) affects both the plasticity of PAP in vivo and of primary cortical astrocytes in vitro (Di Benedetto et al., 2016). Thus, FLX may activate astrocyte-specific factors responsible for such a remodeling capacity. To identify these factors, in this work we performed a microarray screening to profile transcriptomes of astrocytes after acute treatment with FLX and another $A D$, desipramine (DMI) to pick drugdependent effects. We thereby isolated growth/differentiation factor 15 (GDF15) as an early induced target of ADs. GDF15 is a member of the transforming growth factor- $\beta$ (TGF $\beta$ ) superfamily which shows a

\section{Significance}

Astrocytes represent bridging elements between parenchymal and vascular compartments, thereby forming a functional neurovascular unit that might "sense" the brain state and adapt its molecular profile or secrete factors in the bloodstream as a reflection of this state. Thus, astrocyte-specific molecular signatures may become useful biomarkers of distinct cellular (dys)functions in health and disease. In a translational perspective, their characterization in pathological conditions may support the development of diagnostic tools or the selection of tailored treatment options for individual patients.

peculiar expression in the choroid plexus and in astrocytes of the adult rat brain (Strelau et al., 2000) and exerts potent trophic and protective effects on motor neurons (Strelau et al., 2009). Among other members of the same family, GDF10 drives axonal sprouting and recovery from stroke (Li et al., 2015), thereby prompting us to hypothesize that growth/ differentiation factors may additionally affect the sprouting and remodeling of glia cell processes. Here, we explored how GDF15 expression was modulated in cortical astrocytes and PAP of the adult rat PFC upon FLX treatment. We provided a link between FLX-induced cellular plastic changes and GDF15 increased expression. Furthermore, we demonstrated a physiological role of GDF15 in driving astrocyte morphological changes associated with improved BBB properties. Our findings identify an astrocyte-secreted factor, which promotes astrocyte morphological changes and may reverse disease phenotypes accompanied by deficits in glia cell morphology linked to BBB impairments.

\section{2 | MATERIAL AND METHODS}

\section{1 | Animals}

Adult male Wistar rats (10-12 weeks old, 280-350 g, RRID:RGD_13508588) selectively bred for $\mathrm{HAB}$ and weight-matched nonselectively bred rats (NAB) were used in this study (Beiderbeck et al., 2012). Breeding of $H A B$ and $N A B$ animals was performed at the animal facilities of the University of Regensburg. Animals were housed under standard laboratory conditions in groups of four (12 hr light: dark cycle, $22-24^{\circ} \mathrm{C}$, lights on at 06:00 a.m., food and water ad libitum). Animals were assigned randomly to various experimental groups and experimenters were blind to group assignment and outcome assignment. Animal experiments were approved by the government of the Oberpfalz, Germany, and performed in accordance with the European Communities Council Directive of 24 November 1986 (86/609/EEC).

\section{2 | Cell culture}

Rat C6 glioma cells (provided by Prof. Dr. B. Hamprecht; Faculty of Chemistry and Pharmacy; Eberhard-Karls University, Tübingen, 
Germany) were cultured and maintained in DMEM containing $4.5 \mathrm{~g}$ glucose/L, 10\% fetal calf serum (FCS), 1\% penicillin and streptomycin, $1 \mathrm{mM}$ sodium pyruvate (all from GIBCO, Invitrogen, Darmstadt, Germany) at $37^{\circ} \mathrm{C}$ in a humid atmosphere of $5 \% \mathrm{O}_{2} / 95 \% \mathrm{CO}_{2}$. For the microarray experiment, confluent cells were collected and seeded into 6-well plate at a density of $2 \times 10^{5} /$ well in DMEM with FCS. Cell medium was changed to serum-free DMEM $24 \mathrm{hr}$ later prior drug treatment.

Rat primary astrocytes were prepared from PND1 NAB or HAB rats as previously described (Di Benedetto et al., 2016). In brief, brains were isolated, cortices dissected and digested with $0.25 \%$ trypsin containing $1 \mathrm{mM}$ EDTA for $20 \mathrm{~min}(\mathrm{~min})$ at $37^{\circ} \mathrm{C}$ with gentle shaking. The remaining tissue pieces were triturated with fire-polished Pasteur pipettes to yield dissociated cells. Cells were centrifuged at $90 \mathrm{~g}$ for $5 \mathrm{~min}$ and resuspended in DMEM supplemented with 10\% FCS, 2 mM GlutaMAXI, penicillin (100 units/ml), streptomycin $(100 \mu \mathrm{g} / \mathrm{ml})$, and $0.1 \mathrm{mM}$ MEM Non-Essential Amino Acids (Invitrogen, Darmstadt, Germany) on poly-D-lysine-coated $75 \mathrm{~cm}^{2}$ flasks. After reaching $80 \%-90 \%$ confluency, cells were collected and seeded on 6-well or 24-well plates (with glass coverslips) at a density of $3 \times 10^{5}$ or $5 \times 10^{4}$ cells/well, respectively.

Rat brain microvascular endothelial cells (RBMEC) were purchased from Pelo Biotech, Munich. Cells were used at passage 5 to 9. After reaching confluence, cells were seeded in 6-well plate transparent filter inserts ( $1 \mu \mathrm{m}$ pore size, Greiner bio-one, Rainbach im Mühlkreis Austria) or in 24-well plate with cover slips at a density of $1 \times 10^{5}$. Cells were maintained in complete rat EC medium plus supplements (Pelo Biotech) and allowed to reach confluence (approximately $2-3 d$ in culture).

\section{3 | Drug treatments}

For the microarray experiment and its validation by qPCR, cell culture medium was changed to serum-free medium $24 \mathrm{hr}$ prior treatment with $25 \mu \mathrm{M}$ FLX or DMI (Sigma, Taufkirchen, Germany) for $2 \mathrm{hr}$. After treatment, cells were washed with ice-cold PBS, harvested and RNA was prepared using TRI Reagent, following manufacturer's instructions (Sigma).

For experiments with $\mathrm{NAB}$ and $\mathrm{HAB}$ animals, rats received an intraperitoneal injection of either saline or $10 \mathrm{mg} / \mathrm{kg} \mathrm{FLX}$ twice a day for 2 days. This drug concentration is effective in the Forced Swim Test after $24 \mathrm{hr}$ treatment, as predictive of its efficacy (Slattery \& Cryan, 2012). On day 3, animals were anesthetized and transcardially perfused with $4 \%$ paraformaldehyde (PFA, Sigma) in PBS. Brains were removed and post-fixed overnight at $4^{\circ} \mathrm{C}$, cryoprotected in $25 \%$ sucrose in PBS and cut coronally at $40 \mu \mathrm{m}$ on a cryostat. Sections were preserved in PBS containing $25 \%$ ethylene glycol, $25 \%$ glycerol $-20^{\circ} \mathrm{C}$ until further processing for immunofluorescent-immunohistochemistry (IF-IHC). For IFimmunocytochemistry (IF-ICC) experiments, primary astrocytes were treated with $10 \mu \mathrm{M}$ FLX or with different concentrations of human recombinant GDF15 (hrGDF15, 0.01, 0.1, or $1.0 \mathrm{ng} / \mathrm{ml}$ as in (Strelau et al., 2000), R\&D Systems, Wiesbaden-Nordenstadt, Germany) for 48 or $96 \mathrm{hr}$. After treatment, cells were washed with ice-cold PBS, fixed in 4\% PFA for $20 \mathrm{~min}$ at room temperature, washed with PBS and maintained at $4^{\circ} \mathrm{C}$ until further processed. The human recombinant GDF15 was preferred to the rat to perform parallel experiments in human cells, which might be unable to respond to the rat GDF15.

For treatment of astrocytes with the inhibitor of extracellular signal-regulated kinase (ERK) phosphorylation, U0126, cell medium was changed to serum-free medium $24 \mathrm{hr}$ prior a 48-hr treatment with either $20 \mu \mathrm{M}$ U0126 alone, hrGDF15 (1.0 ng/ml) or a combination of both (with U0126 administered 30 min before hrGDF15).

For treatment of RBMEC for IF-ICC or transendothelial electrical resistance (TEER) $24 \mathrm{hr}$ before treatment, cell medium was changed to serum-free medium, followed by a $48 \mathrm{hr}$ application of either hrGDF15 (1.0 ng/ml), astrocyte-conditioned medium (ACM, obtained from confluent NAB astrocyte cultures without FCS) or ACM in which soluble GDF15 was pre-adsorbed with anti-GDF15 antibody for $30 \mathrm{~min}$ at $37^{\circ} \mathrm{C}(500 \mathrm{ng} / \mathrm{ml}$, Abcam, Cambridge, UK). As experimental control, the following compounds were used human recombinant TGF $\beta$ (1 or 5 ng/ml (Boyan et al., 2016; Hu et al., 2016, 2017) or rat recombinant NGF (1 or $10 \mathrm{ng} / \mathrm{ml}$ (Wan et al., 2014)) both purchased from R\&D Systems, Wiesbaden-Nordenstadt, Germany.

\section{4 | Microarray screening}

The microarray screening was performed using the Illumina RatRef-12 Expression BeadChip platform (Illumina Inc., Institute Pasteur, Lille, France). For each treatment condition, biological experiment was analyzed which included six technical replicates. The microarray was performed according to the manufacturer's instructions (Inc.). In brief, after RNA extraction, RNA quality was assessed using the NanoPhotometer (Nanodrop, Implen, Germany) and agarose gel electrophoresis. About $250 \mathrm{ng}$ total RNA was used for in vitro cRNA transcription with the Illumina ${ }^{\circledR}$ TotalPrep ${ }^{\mathrm{TM}}$ RNA Amplification Kit (Ambion, Austin, TX, USA). For the hybridization, 500 ng of cRNA was used. The hybridization was performed in Illumina chambers at $58^{\circ} \mathrm{C} 17.5 \mathrm{hr}$ followed by staining with Cy3-streptavidin. The BeadChip was scanned on a high-resolution Illumina BeadArray reader (Software 3.5.49.29917). The Illumina GenomeStudio software (Version 1.9.0) was used to extract the expression data (fluorescence intensities; Dunning, Smith, Ritchie, \& Tavare, 2007; Huber, von Heydebreck, Sultmann, Poustka, \& Vingron, 2002). Normalization and differential expression analyses were performed in R using the beadarray, vsn, and limma packages (R Core Team, 2015; Smyth, 2004).

\section{5 | Candidate gene selection}

Candidate genes were selected for further validation according to maximal upregulation ( $\geq 2.5$-fold for $F L X$ and $\geq 2$-fold for DMI) or 
downregulation ( $\geq-1.5$-fold for both drugs), based on the corresponding single contrast analysis and appearance in the GO clusters. Moreover, appearance of genes in both FLX- and DMI- modulated targets was used as a third selection criterion. Following these criteria, seven genes were included in the further validation.

\section{6 | Validation of microarray data with qRT-PCR (qPCR)}

After drug treatments, cells were collected and RNA was prepared as mentioned above. Concentration was measured using NanodropPhotometer (NanoPhotometer ${ }^{\circledR}$, Implen, Munich, Germany). A maximum of $1 \mu \mathrm{g}$ total RNA was digested with DNase (RQ1 RNase-Free DNase Kit, Promega, Mannheim, Germany) followed by reverse transcription using Superscript II (Invitrogen, Karlsruhe, Germany). The cDNA was analyzed by qPCR using the QuantiFastSYBR Green room temperature-PCR Kit (Qiagen, Hilden, Germany). Oligonucleotide primers were custom-designed by Sigma. Primer efficiencies were determined once for the whole study. Experiments were performed in duplicates on the LightCyclers system 2.0 (Roche Diagnostics, Mannheim, Germany) with the following protocol: a preincubation step at $95^{\circ} \mathrm{C}$ for $5 \mathrm{~min}$ fol lowed by denaturation at $95^{\circ} \mathrm{C}$ for $10 \mathrm{~s}$ and final amplification step at $60^{\circ} \mathrm{C}$ for $30 \mathrm{~s}$ for 40 cycles. At the end of every run, a melting curve was recorded $\left(42-60^{\circ} \mathrm{C}\right.$ with $\left.20^{\circ} \mathrm{C} / \mathrm{s}\right)$. The $C_{P}$ values were determined using the maximum of the second derivative of the amplification curve as threshold line. Relative gene expression was calculated following (Pfaffl, 2001):

$$
\text { ratio }=\frac{E_{\text {target }}^{\Delta C t[\text { control }- \text { sample }]_{\text {target }}}}{E_{\text {house keeper }}^{\Delta C t[\text { control }- \text { sample }]_{\text {house keeper }}}}
$$

\section{7 | Fluorescent in situ hybridization}

\subsection{1 | Generation of the gdf15 probe}

PCR oligonucleotides were designed complementary to bases 263-622 of the rat sequence of gdf15 mRNA NM_019216.2 (5'-GGA TACTCAGTCCAGAGGTGAGA-3' and 5'-CTGTCCTGTGCATAAGAAC CAC $\left.-3^{\prime}\right)$. PCR was performed on cDNA prepared from RNA extracted from rat primary astrocytes and amplified fragments were cloned using a Strata Clone PCR Cloning Kit (Stratagene, Waldbronn, Germany). To generate the ISH probe, DNA was linearized and transcribed with Maxiscript T7/T3 RNA labeling kit (Ambion ${ }^{\circledR}$, Invitrogen) following the manufacturer's instructions. Riboprobes were purified with Mini Quick Spin RNA columns (Roche, Penzberg, Germany; Tanasic et al., 2016).

\subsection{2 | Brain slices preparation and fluorescent in situ hybridization procedure}

Slices were washed with $2 \times S S C(0.3 \mathrm{M}$ sodium chloride, $30 \mathrm{mM}$ sodium citrate, $\mathrm{pH}$ 7.0, Sigma), before proceeding to fluorescent in situ hybridization. In brief, they were pretreated with acetic anhydride (Sigma) solution, incubated in ice-cold methanol/acetone (1:1), and pre-hybridized in ready-to-use pre-hybridization solution (Sigma) in a humid chamber while shaking at room temperature. Then, the RNA probe was diluted in hybridization buffer (Sigma), denatured and added to slices. Incubation proceeded overnight at $56^{\circ} \mathrm{C}$ while shaking. Slices were then treated with RNase A (Roche) at $37^{\circ} \mathrm{C}$ and with $3 \% \mathrm{H}_{2} \mathrm{O}_{2}$ to quench endogenous peroxidases. Afterward, they were blocked in Tris- $\mathrm{NaCl}$ buffer (TNB) blocking reagent (Roche) at room temperature and incubated overnight with anti-DIG HRP (Roche) in TNB. The day after, they were incubated in Cy3-conjugated tyramine in amplification diluent (Roche) together with DAPI 1:1,000, washed and mounted on slides with anti-fading mounting medium (Aquapolymount, Polysciences Europe $\mathrm{GmbH}$, Eppelheim, Germany) for confocal imaging.

\subsection{3 | RNA interference (RNAi), cell transfection, and drug treatment}

Short interfering RNAs (siRNAs) complementary to the rat mRNA sequence coding for GDF15 were synthesized (Dharmacon Research, Lafayette, CO). Two different siRNAs (si1, 5'-ACUCAACCCGGACGAGCUAUU-3' and si2, 5'-UGACCCAGCUGUCCGGAUAUU-3') were selected together with a scrambled siRNA (scr, 5'-CCUAAGGUUAAGUCGCCCUUU-3'), which was used as negative control. All sequences were submitted to a BLAST search to verify their specific targeting of gdf15 mRNA (si1 and si2) and the lack of targeting of any sequence for scr RNA. Transfections were performed using Lipofectamine ${ }^{\mathrm{TM}} 2000$ (Invitrogen), following the manufacturer's instructions. In brief, $24 \mathrm{hr}$ before transfection, medium was changed to medium without antibiotics. Each siRNA was administered at a final working concentration of 50 or $100 \mathrm{nM}$ to test its efficiency. After 3 days, FLX was administrated as described above. Then, cells were washed with PBS, fixed and maintained at $4^{\circ} \mathrm{C}$ until further processed for IF-ICC.

For treatments with $\mathrm{ACM}$, supernatants from different treatment conditions were collected and directly transferred on $\mathrm{HAB}$ astrocytes, with the exception of antibody-treated ACM for $30 \mathrm{~min}$ at $37^{\circ} \mathrm{C}$ (with 100 and $500 \mathrm{ng} / \mathrm{ml}$ of anti-GDF15 antibody).

\subsection{4 | Immunofluorescent- immunohistochemistry and immunocytochemistry}

For IF-IHC in rat brains, sections were washed thoroughly before permeabilization and blocking in 0.1\% Triton-X 100+2\% Normal Goat Serum (NGS, Vector Labs, Biozol, Eching, Germany) in PBS for $2 \mathrm{hr}$ at room temperature. Then, they were incubated with mouse anti-GFAP antibody (1:400, Sigma, Cat\#G3893, RRID:AB_477010) and goat antiGDF15 antibody (1:200, Abcam, Cat\#ab39999, RRID:AB_732535), in $0.1 \%$ TX $100+2 \%$ NGS in PBS overnight at $4^{\circ} \mathrm{C}$, followed by incubation with anti-mouse-Cy3 (1:400, Invitrogen, Cat\#C2181, 
RRID:AB_258785), anti-goat Alexa Fluor 488 (1:400, Invitrogen, Cat\#A-11001, RRID:AB_2534069) and DAPI (1:1,000, Sigma) in 2\% NGS in PBS for 2 hr at room temperature. Finally, all sections were washed and mounted on slides for confocal analysis. For Figure 4, brain slices underwent the same procedure as described above, except the following antibodies were used mouse anti-GFAP (1:400), rabbit antiClaudin-5 (Cln5 1:250, Sigma, Cat\#SAB4502981, RRID:AB_10753223) as secondary antibodies anti-mouse Cy3 (1:400), anti-rabbit AlexaFluor 488 (1:1,000), and DAPI (1:1,000) were used.

For IF-ICC on primary astrocytes, cells were permeabilized and blocked in $0.2 \%$ Triton-X $100+2 \%$ NGS in PBS for $1 \mathrm{hr}$ at room temperature. Then, they were incubated overnight at $4^{\circ} \mathrm{C}$ with mouse anti-GFAP + mouse anti-S100ß (1:400 and 1:1,000, respectively, Sigma; S100ß: Abcam, Cat\# ab11178, RRID:AB_297817) in 1\% NGS in PBS. Cells were washed and incubated with anti-mouse-Cy3 (1:200) and DAPI $(1: 1,000)$ in $1 \%$ NGS in PBS $1 \mathrm{hr}$ at room temperature. After washing, coverslips were mounted on slides for confocal microscopy.

For IF-ICC in RBMVEC, after treatment, cells were washed with ice-cold PBS and fixed in methanol $\left(-20^{\circ} \mathrm{C}\right.$, for $\left.20 \mathrm{~min}\right)$; cells were then permeabilized and blocked with $0.2 \%$ Triton-X $100+3 \%$ NGS in PBS for $1 \mathrm{hr}$ at room temperature. Then, they were incubated overnight at $4{ }^{\circ} \mathrm{C}$ with rabbit-Claudin 5 (1:200) in $0.2 \%$ Triton-X $100+3 \%$ NGS in PBS. Cells were washed and incubated with anti-rabbit AlexaFluor 488 (1:400) and DAPI (1:1,000) in 2\% NGS in PBS $2 \mathrm{hr}$ at room temperature. After washing, coverslips were mounted on slides for confocal microscopy.

\subsection{5 | GDF15 release enzyme-linked immunosorbent assay}

After drug treatments, conditioned media were collected and GDF15 protein levels were determined using a GDF15 enzyme-linked immunosorbent assay (ELISA) Kit (R\&D Systems), following the manufacturer's instructions.

\subsection{6 | Western blot procedure}

After transfections or drug treatments, cells were washed in ice-cold PBS. They were homogenized in a buffer containing $250 \mu \mathrm{M}$ Tris$\mathrm{HCl}, \mathrm{pH} 7.5$, and protease inhibitor cocktail (Roche) and lysed in RIPA buffer overnight at $4^{\circ} \mathrm{C}$. Then, total proteins for each sample were denatured in Laemmli buffer, separated with 10\% SDS-PAGE and transblotted to a nitrocellulose membrane (Protran BA, Whatman, GE Healthcare, Munich, Germany). Membranes were blocked in Tris-buffered saline with Tween-20 (TBST) with 5\% bovine serum albumin (BSA) overnight at $4^{\circ} \mathrm{C}$. Thereafter, they were incubated for $2 \mathrm{hr}$ at room temperature with goat anti-GDF15 (1:250) or a mouse anti- $\beta$ actin (1:4,000, CellSignaling, Cat\#3700, RRID:AB_2242334) as standard internal control for semi-quantitative analysis. Blots were washed in TBST and incubated with HRP-conjugated donkey antigoat (1:2,500, Santa Cruz Biotechnology, Santa Cruz, CA, USA) and rabbit anti-mouse (1:10,000, Dianova, Hamburg, Germany) for $1 \mathrm{hr}$ at room temperature. After washing, they were visualized with enhanced chemiluminescence (ECL) detection reagent (GE Healthcare) and films were scanned and processed by densitometric analysis using ImageJ software (Rasband, W.S., ImageJ, U. S. National Institutes of Health, Bethesda, USA, http://imagej.nih.gov/ij/, RRID:SCR_003070).

\subsection{7 | Confocal laser scanning microscopy}

Confocal microscopy was performed at room temperature with a confocal laser scanning microscope (inverted type IX81, Olympus Europe Holding $\mathrm{GmbH}$, Hamburg, Germany) equipped with an UAPO 40×/340 NA 1.15 water-immersion objective (ZEISS) using the FluoView FV1000 software (Version 2.1c; Olympus FluoView Resource Center). Excitation was provided by lasers of 405, 488, $559 \mathrm{~nm}$ wavelength (Visitron Systems). For experiments in adult brains and cell culture (fluorescent in situ hybridization, IF-IHC, IFICC), images were acquired from at least two slices per each brain (10 images/slice, with an average of 20 optical sections, $1 \mu \mathrm{m}$ Z-step size, per image) or 10-15 cells per experiment (eight optical sections, $1 \mu \mathrm{m}$ Z-step size, per image). Images were converted using the FluoView FV1000 software from the existing proprietary format. oib into the tagged image file format (.tiff) for analysis with Adobe Photoshop CS3 (Adobe Systems, San Jose, California, USA) or into 8-bit.bmp files for analysis with NeuronJ, a plugin of ImageJ (http:// www.imagescience.org/meijering/software/neuronj/).

For analysis of TJ between cells, Fiji ROI manager was used. In brief, five to ten cells per image were surrounded using the polygon selection tool then mean intensity of claudin- 5 staining was measured and used for analysis. To mitigate bias in counting, pictures were taken by an experimenter blind to treatment and analyzed by a second experimenter.

\subsection{8 | Analysis of gdf15 expression}

For the analysis of fluorescent in situ hybridization experiments, the histogram function of Adobe Photoshop CS3 was used, after marking the boundaries between parenchyma and BVs (region of interest, ROI) with the "lasso" tool. The intensity of signal in the red channel (corresponding to gdf15 expression) and the number of pixels in the ROI were recorded for each image. Values used for statistical analysis were obtained after normalizing signal intensity on the ROI in pixels, averaged per animal.

\subsection{9 | Analysis of processes}

For the analysis of $g d f 15^{+}$and $\mathrm{GFAP}^{+}$processes, images were loaded into Fiji's cell counter plugin, processes which were positive for GFAP in the red channel staining which crossed the boundary of the $\mathrm{BV}$ were manually counted. Among those, the ones that are also 
positive for gdf15/GFAP staining in the green channel were additionally quantified and recorded.

For morphological analysis of primary astrocytes, the program NeuronJ was used to count the number of processes per cell and measure their sizes, after converting images from the confocal microscope into 8-bit color pictures compatible with the program.

\subsubsection{0 | Transendothelial electrical resistance measurements}

TEER was measured using the Ohm"s law method (Chen, Einspanier, \& Schoen, 2015; Srinivasan et al., 2015). In brief, the two measuring electrodes were placed across the inner/outer sides of the filter insert to get in contact with the internal and external media, respectively. The Ohm's resistance was obtained by measuring the blank (insert without cells) resistance $\left(R_{\mathrm{BLANK}}\right)$ and measuring the resistance across the cell layer on the filter upon different treatments $\left(R_{\text {TOTAL) }}\right.$. The cell layerspecific difference $\left(R_{\text {CELLS }}\right)$ was calculated using the following formula:

$$
R_{\text {(CELLS) }}(\Omega)=R_{\text {(TOTAL) }}-R_{\text {(BLANK) }} .
$$

To integrate the complete surface areas of the filter insert the following formula was applied:

$$
\operatorname{TEER}_{(\text {REPORTED) }}=R_{(\text {CELLS) }}(\Omega) \times \text { surface area }\left(\mathrm{cm}^{2}\right)
$$

At the end of TEER measurements, filters were fixed in 4\% PFA and stored until further processing.

\section{8 | Statistical analysis}

For all data analyses, we first verified the normality of the distributions to choose the appropriate statistic tool to use. Then, data were analyzed with the unpaired Student's $t$ test when two samples were compared. For multiple comparisons, a one-way ANOVA, a twoway ANOVA, or the nonparametric Kruskal-Wallis test was used. Different post hoc corrections were used: Dunnett's for comparisons of all means against the control mean; Sidak's or Tukey's, for selective means comparisons or comparisons of all means against each other, respectively (for parametric tests); Dunn's for comparisons of all means against each other for nonparametric tests; Fisher's LSD was used when we were interested in few sensible comparisons, as part of the experimental design. In particular, we used it to examine and interpret ex vivo studies, in which the low number of animals available for our experiments might have biased our interpretation of data. Unfortunately, especially the HAB rats were not breeding that well, preventing us to increase their numbers easily. Differences were considered significant when $p \leq 0.05$. Statistical analyses were performed with Prism GraphPad program (GraphPad Prism 6 Software, Inc., La Jolla, CA, USA).

\section{3 | RESULTS}

\subsection{GDF15 expression increases in C6 glioma cells and primary astrocytes upon treatment with antidepressants}

To identify molecular cues triggering astrocyte morphological changes, we screened early induced transcriptome profiles of rat C6 glioma cells, a model of astrocytes, upon treatment with the ADs FLX and DMI. Data discussed in this publication are deposited in NCBI's Gene Expression Omnibus and are accessible through GEO Series accession number GSE89873 (https://www.ncbi.nlm.nih gov/geo/query/acc.cgi?acc=GSE89873). The validation of selected candidates with qPCR in $\mathrm{C} 6$ cells and primary cortical astrocytes revealed a selective increased expression of gdf15 by both ADs in primary astrocytes, though FLX alone additionally modulated the expression of bhlhb2 (Figure 1a, Kruskal-Wallis test $p=0.0001$ followed by Dunn's test; Figure 1b, Kruskal-Wallis test $p=0.0001$ followed by Dunn's test; Table 1 for statistic details).

The further examination of gdf15 expression patterns in acute brain slices upon FLX administration indicated its presence in the choroid plexus, as previously published (Strelau et al., 2000; Figure S1, ii), but also in filamentous structures around BVs of the PFC (Figure S1, iv). The latter results suggested an enrichment of gdf15 in glia cell processes apposed to BV, where the BBB develops.

To get functional insights into the physiological roles of GDF15 in healthy brains, we first injected adult Wistar rats (here called nonselectively bred for anxiety-like behavior (NAB) to distinguish them from $\mathrm{HAB}$ rats used later in this study) with FLX. The examination of gdf15 distribution at BV locations in the PFC revealed that FLX doubled the intensity of gdf15 signal (Figure 1c,d; unpaired Student's $t$ test, $\left.t_{(7)}=3.46, N=4-5, p=0.0105\right)$. We additionally confirmed its enhanced accumulation in filamentous structures extending from parenchymal cells to wrap around BVs (white arrow in Figure 1c, iv), which resembled PAP and suggested a putative effect of gdf15 on this subcellular compartment.

\subsection{GDF15 expression increases in PAP concurrently with their morphological changes upon FLX treatment}

To evaluate the possibility that GDF15 affected PAP, we compared the expression of GDF15 mRNA and protein in astrocytes of the PFC between NAB brains and brains from an animal model of MDD, the HAB rat, which shows an aberrant astrocyte morphology around BVs (Di Benedetto et al., 2016), before and after FLX administration. Using double fluorescent in situ hybridizationIHC and double fluorescent-IHC to label gdf15/GDF15 together with an antibody against glial fibrillary acidic protein (GFAP), we detected a reduced density of $\mathrm{BV}$-associated astrocyte processes $\left(\right.$ GFAP-positive, GFAP $^{+}$) in HAB brains. Interestingly, FLX did not 
(a)

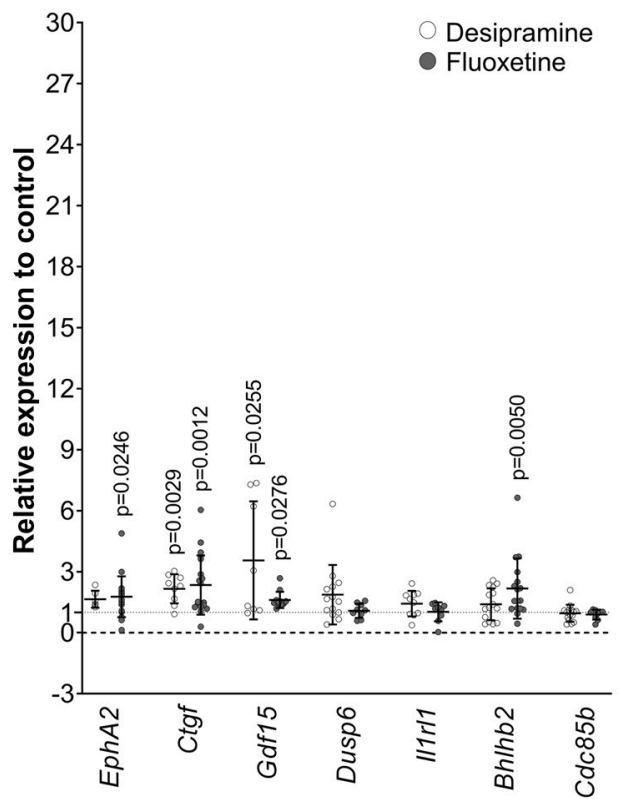

(c)
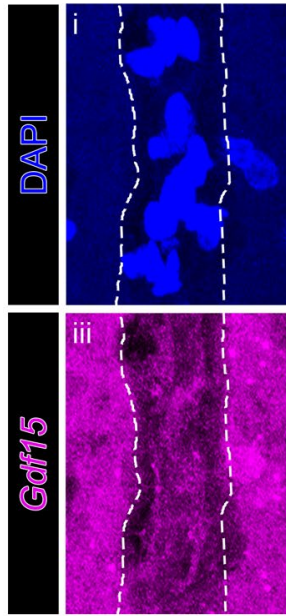

Rat C6 glioma cells
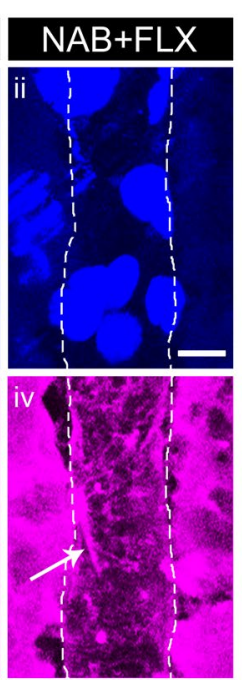

(b)

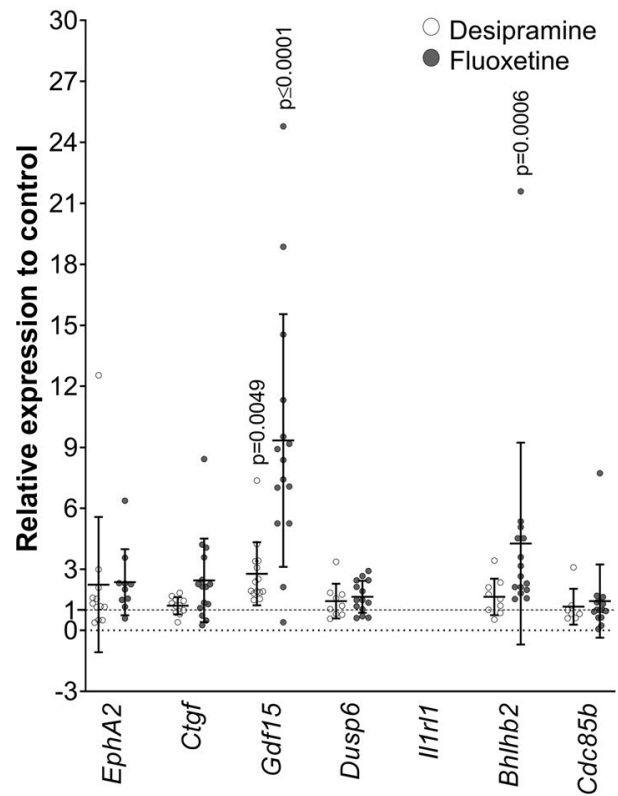

(d)

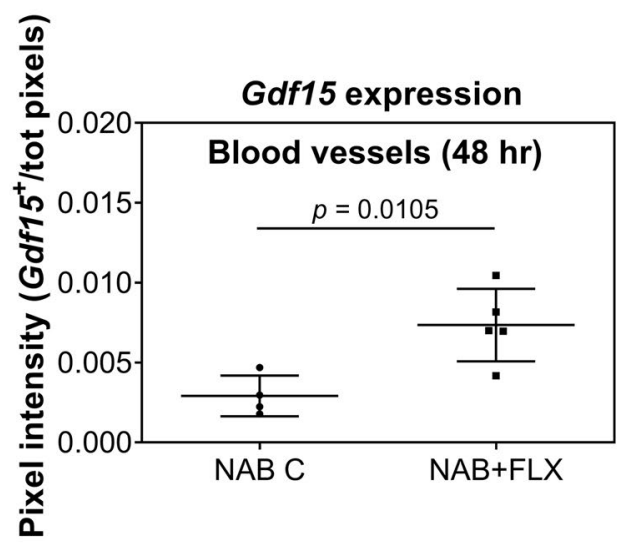

FIGURE 1 FLX and DMI increase gdf15 expression in C6 glioma cells, primary rat astrocytes and in processes between parenchymal cells and blood vessels (BVs) of the prefrontal cortex (PFC). (a-b) A microarray screening on C6 cells show a drug-dependent increased expression of several transcripts shortly ( $2 \mathrm{hr}$ ) after treatment with FLX or DMI (a); the validation of microarray results by qPCR in cortical rat primary astrocytes confirmed gdf15 as a dual drug-specific target (b) ( $N \geq 6$ experiments). (c) Representative images of cortical brain slices derived from saline- or FLX-treated NAB rats depict BVs labeled with DAPI to stain single nuclei (in blue, $\mathrm{i}$ and ii) and with a probe against gdf15 mRNA (dashed lines; in magenta, iii and iv). The white arrow indicates an example of filamentous structures between parenchymal cells and BV, which stained positive for gdf15 (iv). (d) Quantification of gdf15 labeling intensity (in pixels, gdf15 ${ }^{+}$) normalized to the total volume of each BV (total pixels) after 48 hr FLX treatment (NAB C, $N=4$ brains; NAB + FLX, $N=5$ brains). Scale bar, $50 \mu$ m. Data information: data are presented as mean $\pm S D$; in A, Kruskal-Wallis test $p=0.0001$ followed by Dunn's test; in $\mathrm{B}$, Kruskal-Wallis test $p=0.0001$ followed by Dunn's test; in D, Student's $t$ test, $p=0.0105$. Table 1 for statistic details [Color figure can be viewed at wileyonlinelibrary.com]

exert any effects in NAB brains, although it completely restored the density of GFAP ${ }^{+}$processes in HAB brains (Figure $2 a$, b, oneway ANOVA, $F_{(3,8)}=4.222, N=3, p=0.0459$ followed by post hoc Fisher's LSD test; Figure 2d,e, one-way ANOVA, $F_{(3,8)}=5.453$, $N=3, p=0.0246$ followed by post hoc Fisher's LSD test; Table 1). To test whether gdf15/GDF15 modulation was restricted to the glia cell process compartment, we then quantified changes in the density of co-labeled $g d f 15^{+}\left(\mathrm{GDF} 15^{+}\right) / \mathrm{GFAP}^{+}$processes along BVs in the same brains. This analysis revealed a decreased density of co-labeled processes in HAB compared to NAB brains, which was significant when examining protein localization, but not when considering only mRNA differences (Figure 2a,c, one-way ANOVA, $F_{(3,8)}=9.746, N=3, p=0.0048$ followed by post hoc Fisher's LSD test; Figure 2d,f, one-way ANOVA, $F_{(3,8)}=13.83, N=3, p=0.0016$ followed by post hoc Fisher's LSD test; Table 1). However, differences in GDF15 concentration upon drug treatment did not 


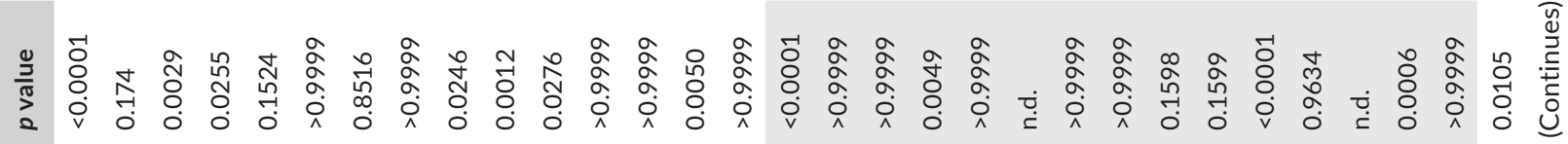

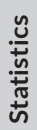

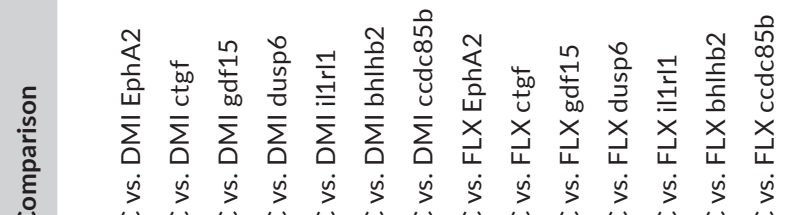

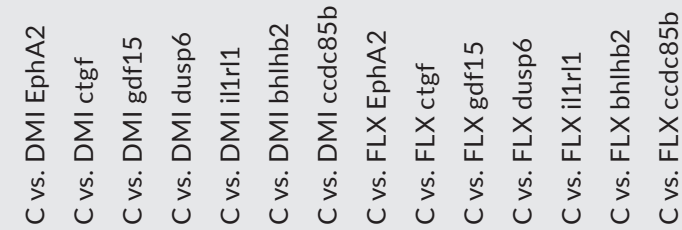

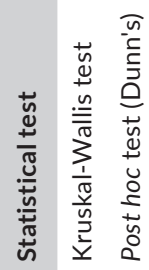

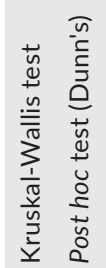

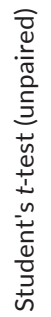

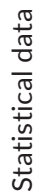
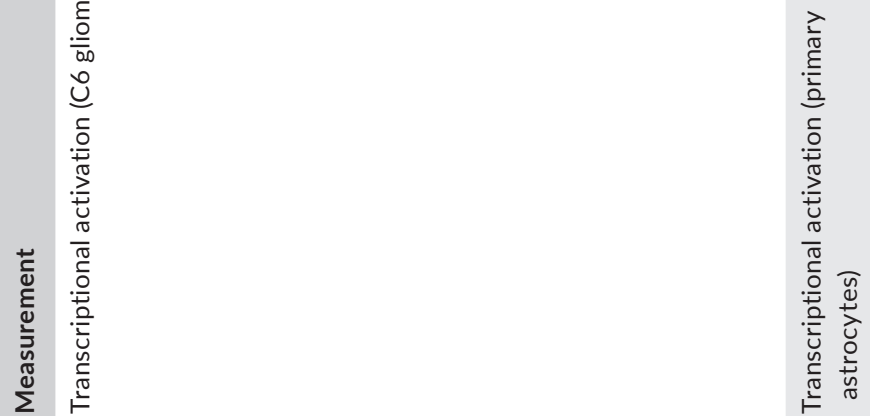

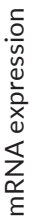

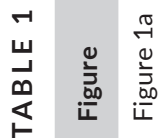

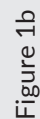

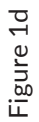




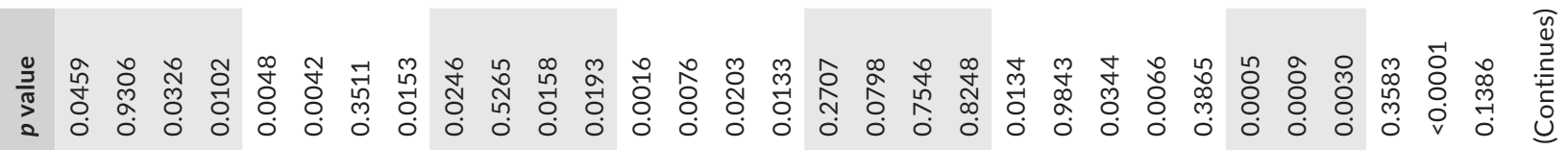
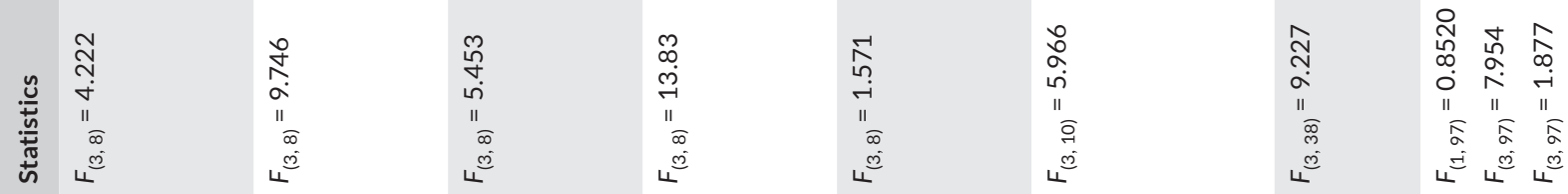

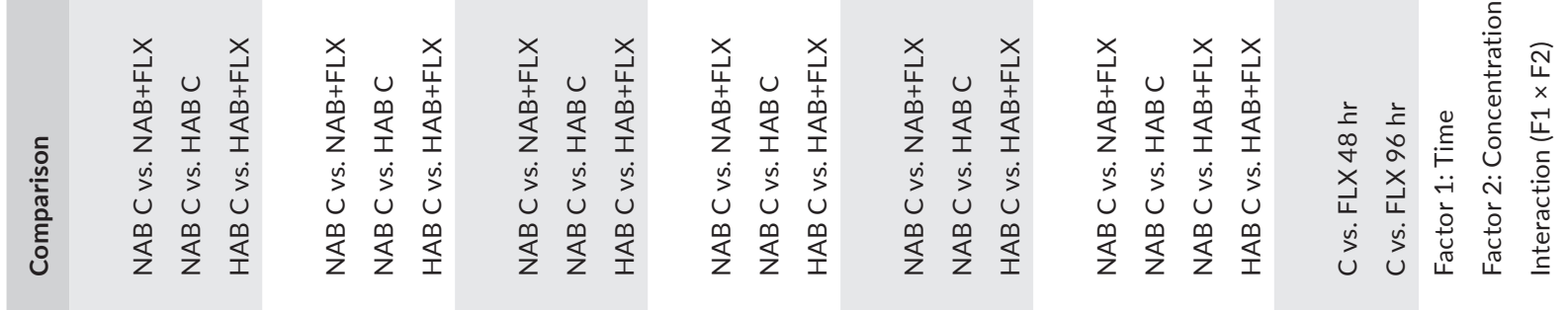

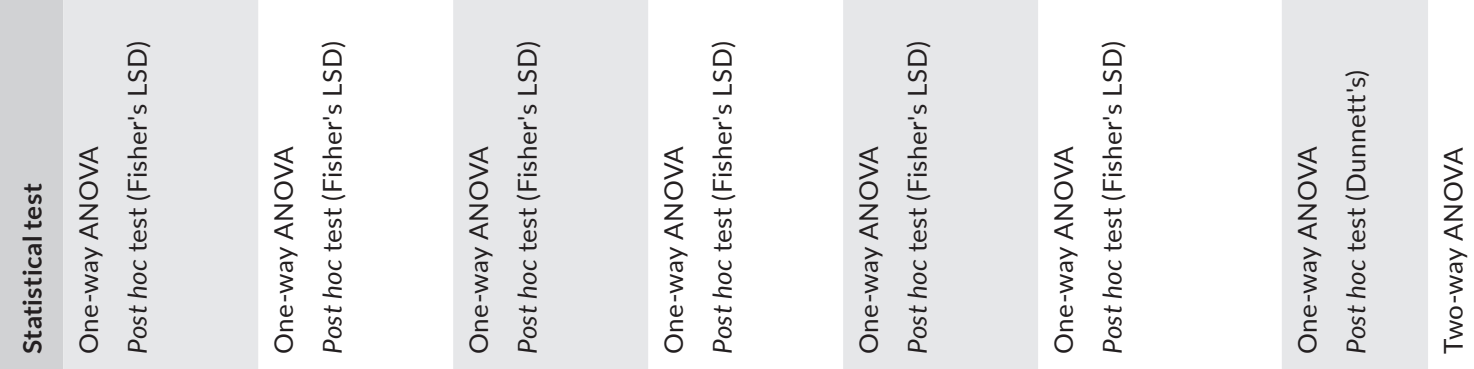

它
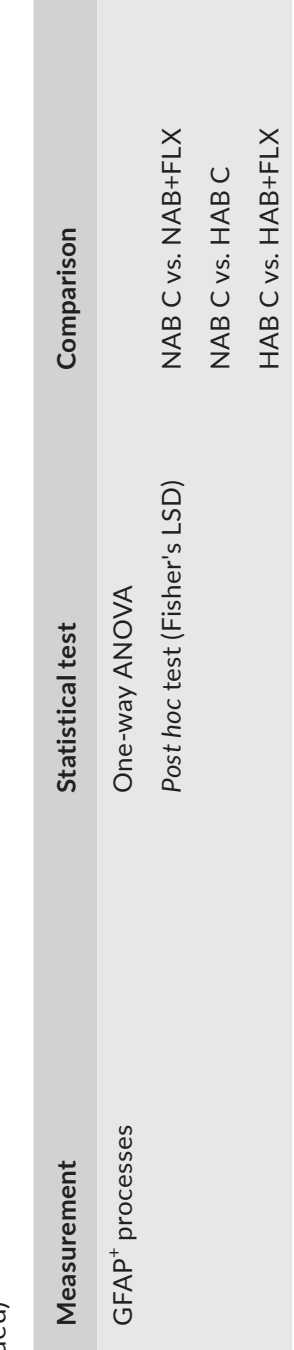

o :
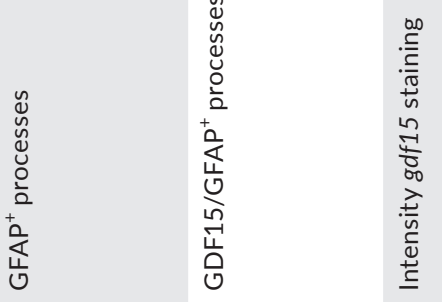

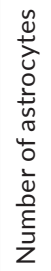

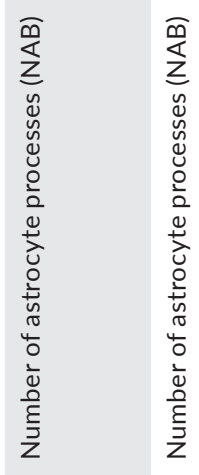

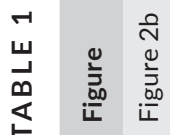

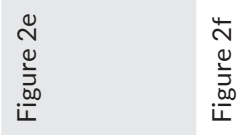

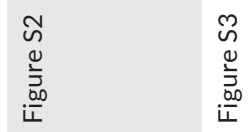

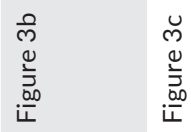




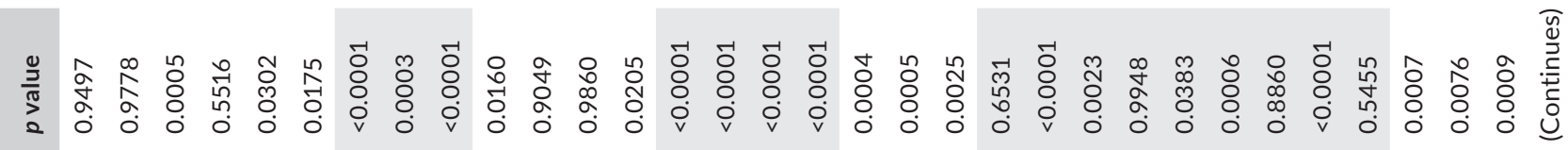

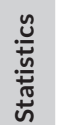
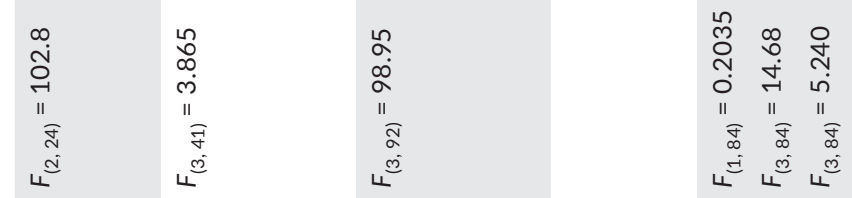

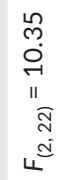

京

它

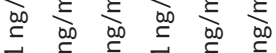

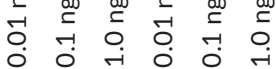

con

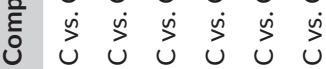

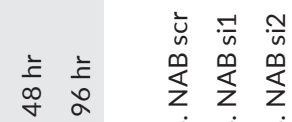

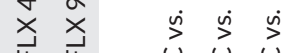

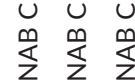

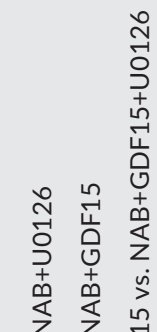

옴ำ

崩空峑

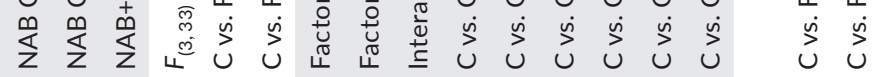

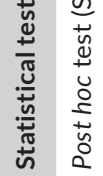
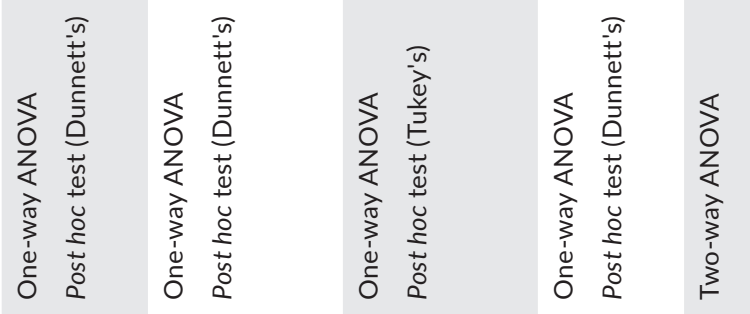

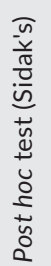
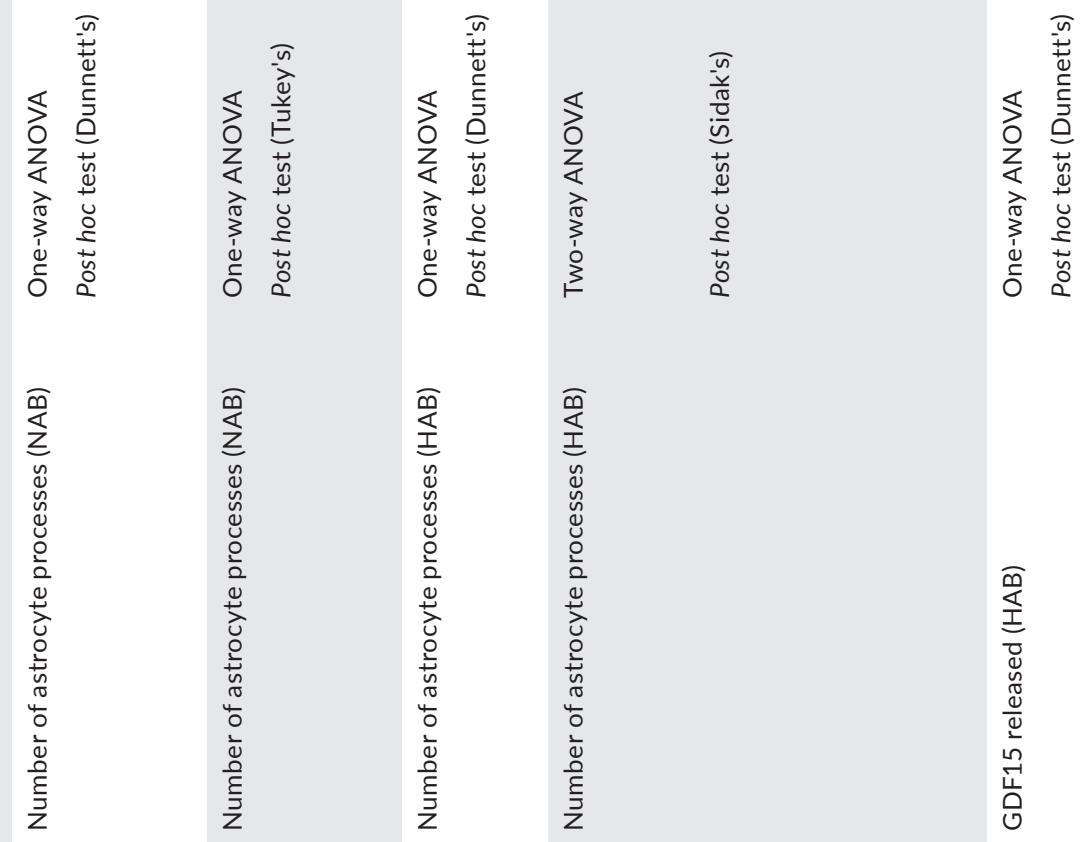

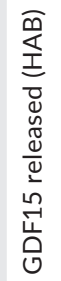

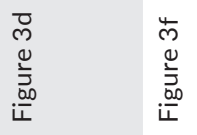

$\frac{5}{m}$
0
$\frac{0}{2}$
$\frac{01}{4}$

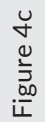

守 


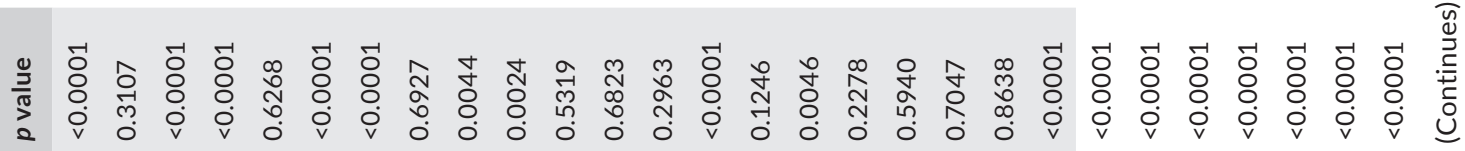

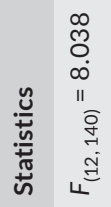

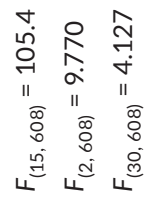

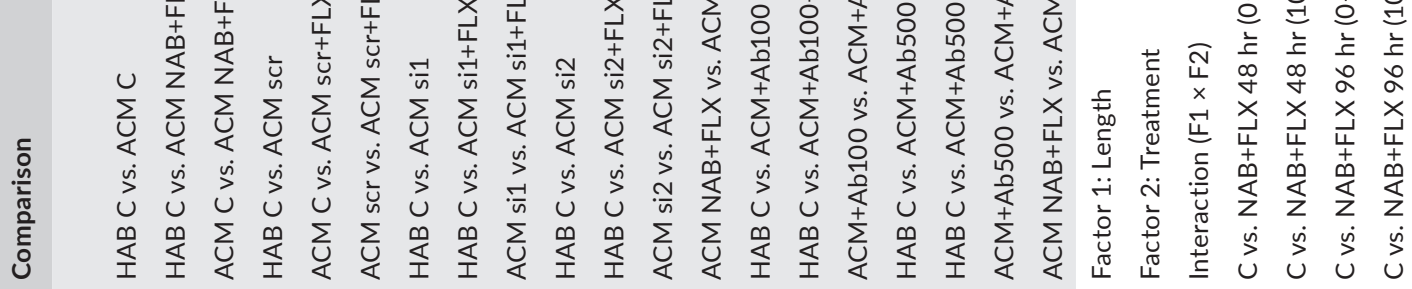

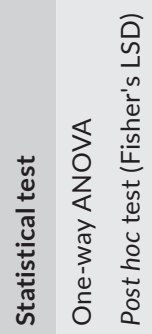

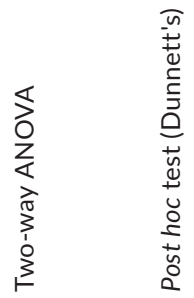

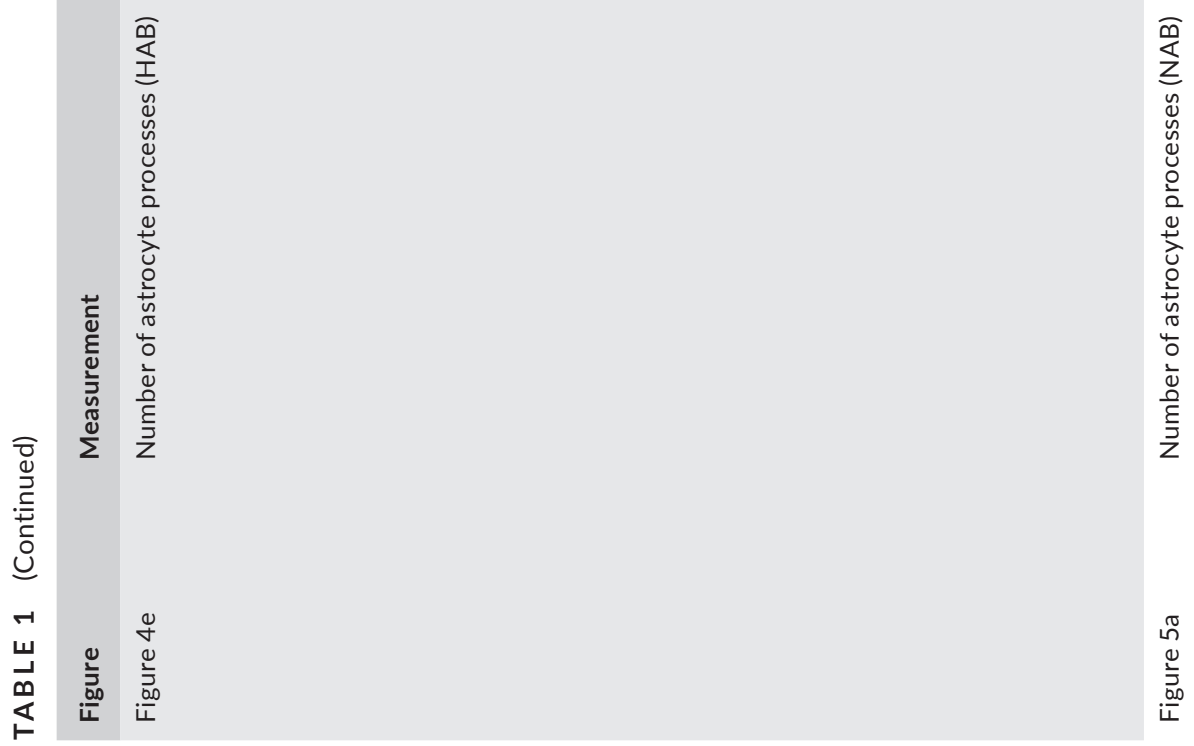




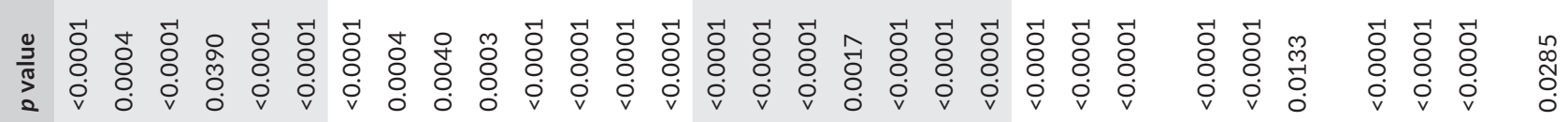

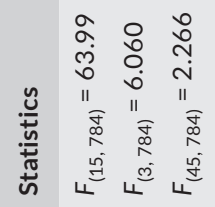
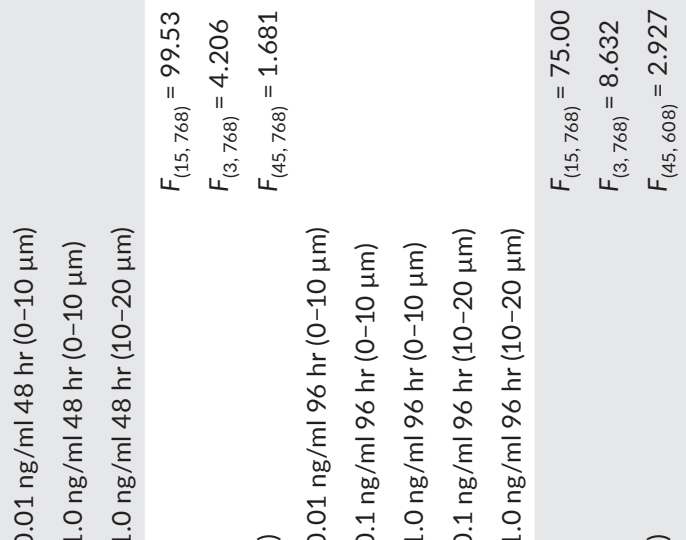

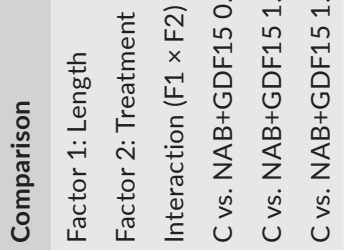

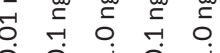
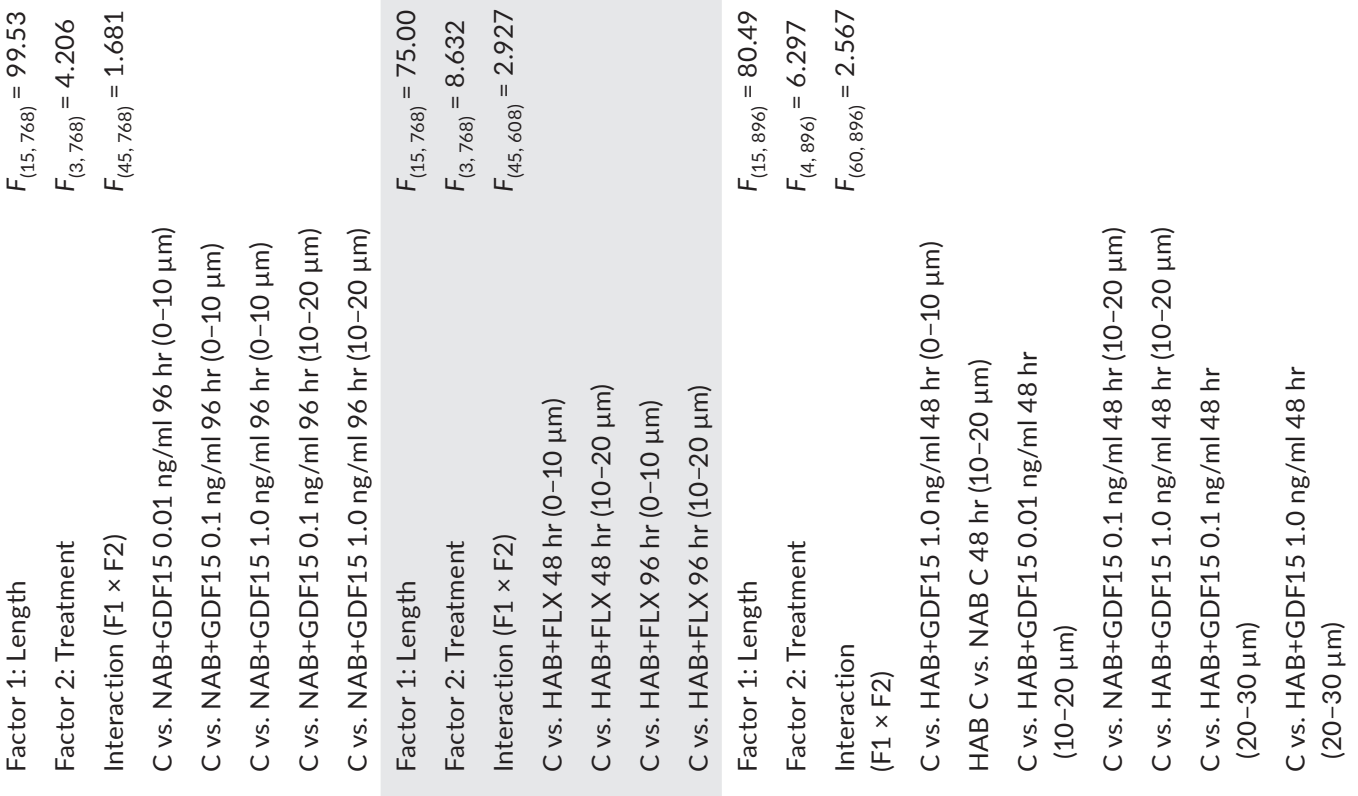
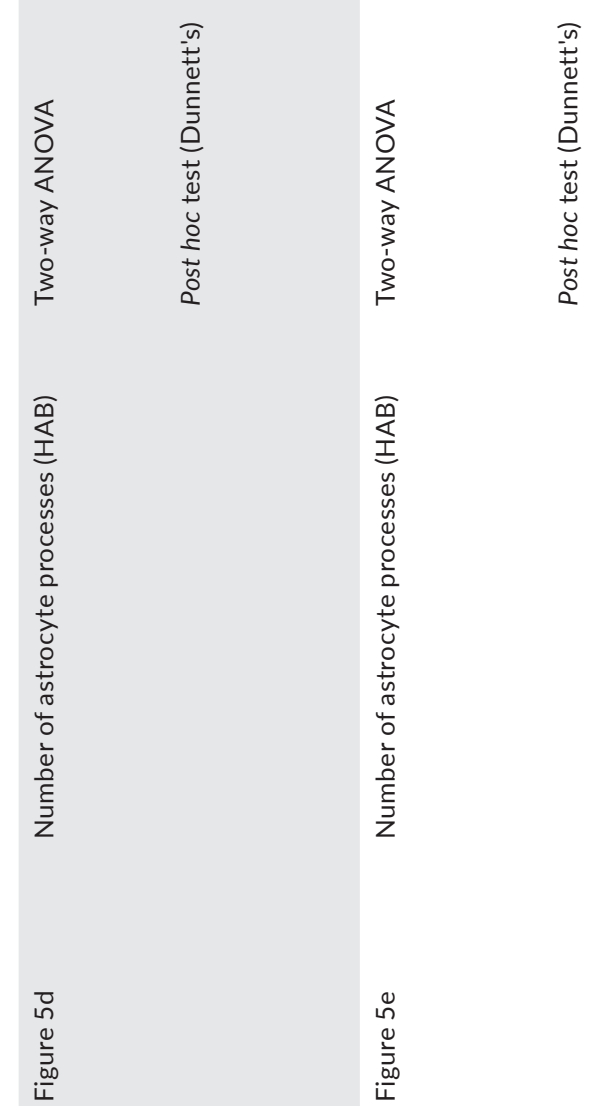

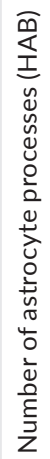

悹

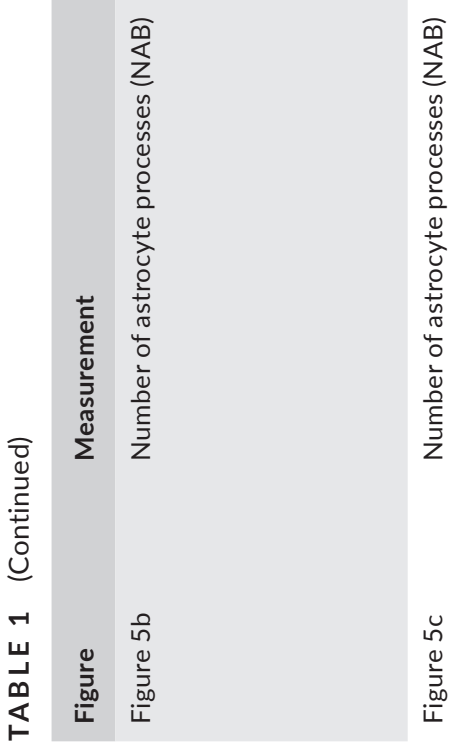




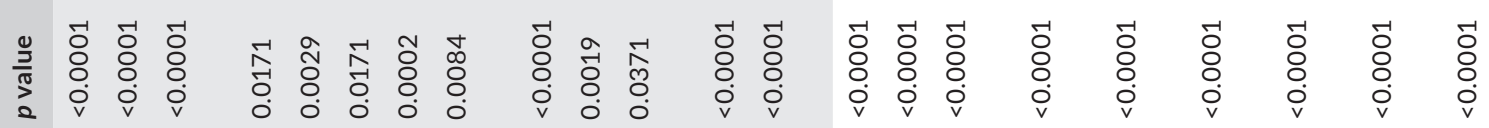

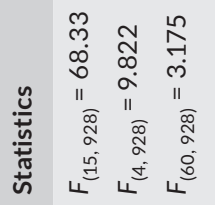

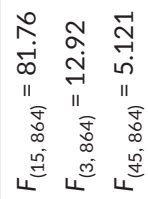
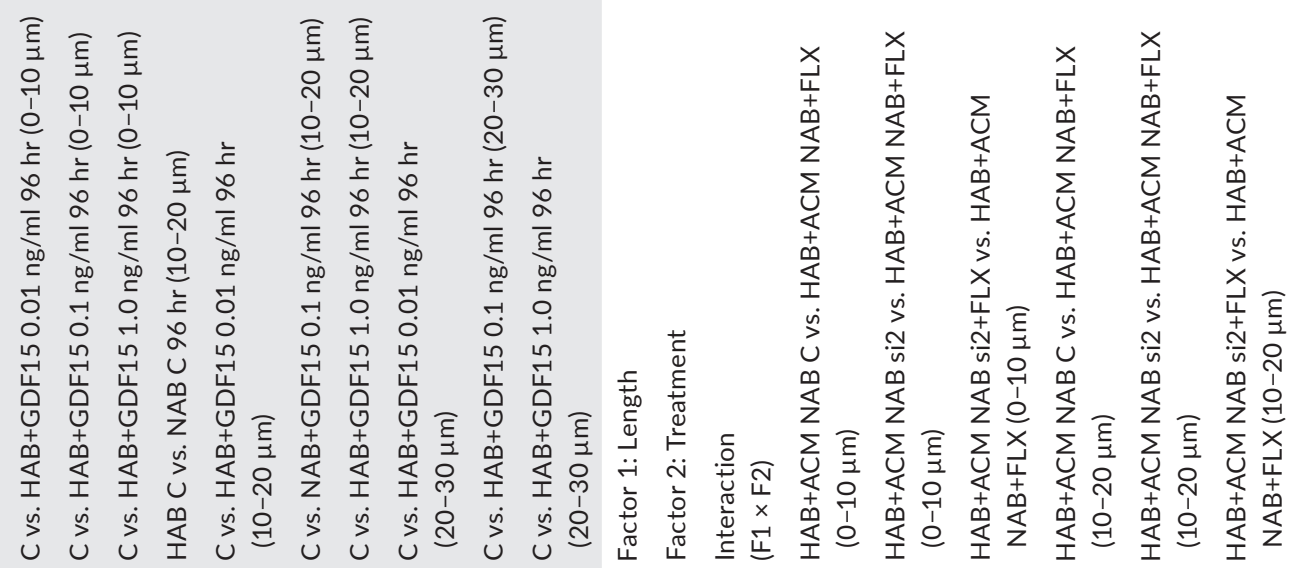

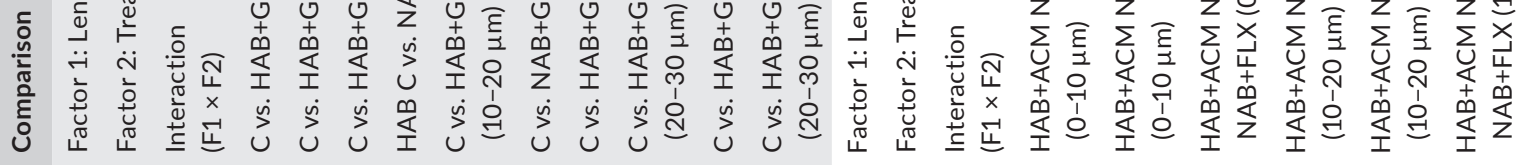
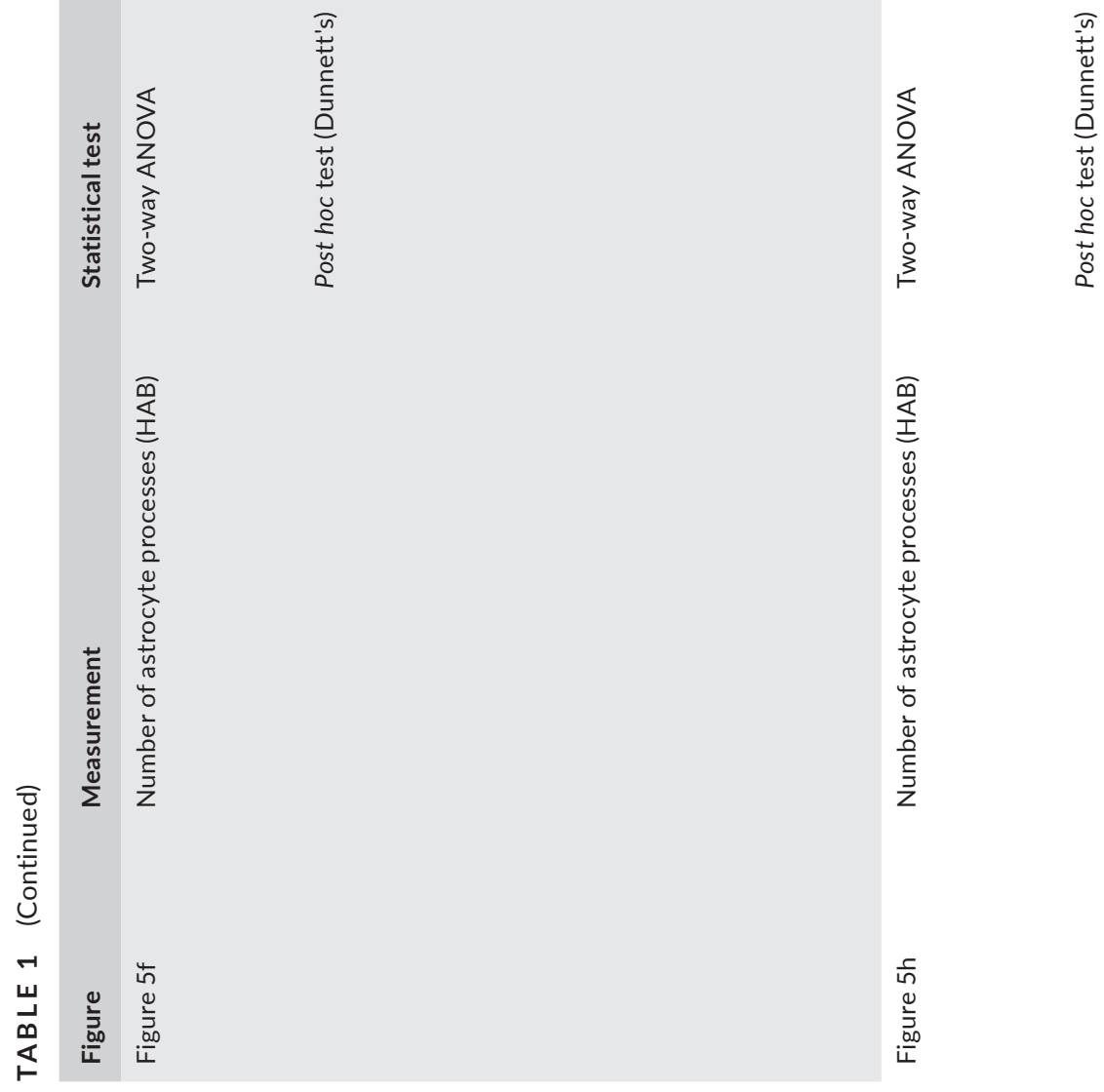


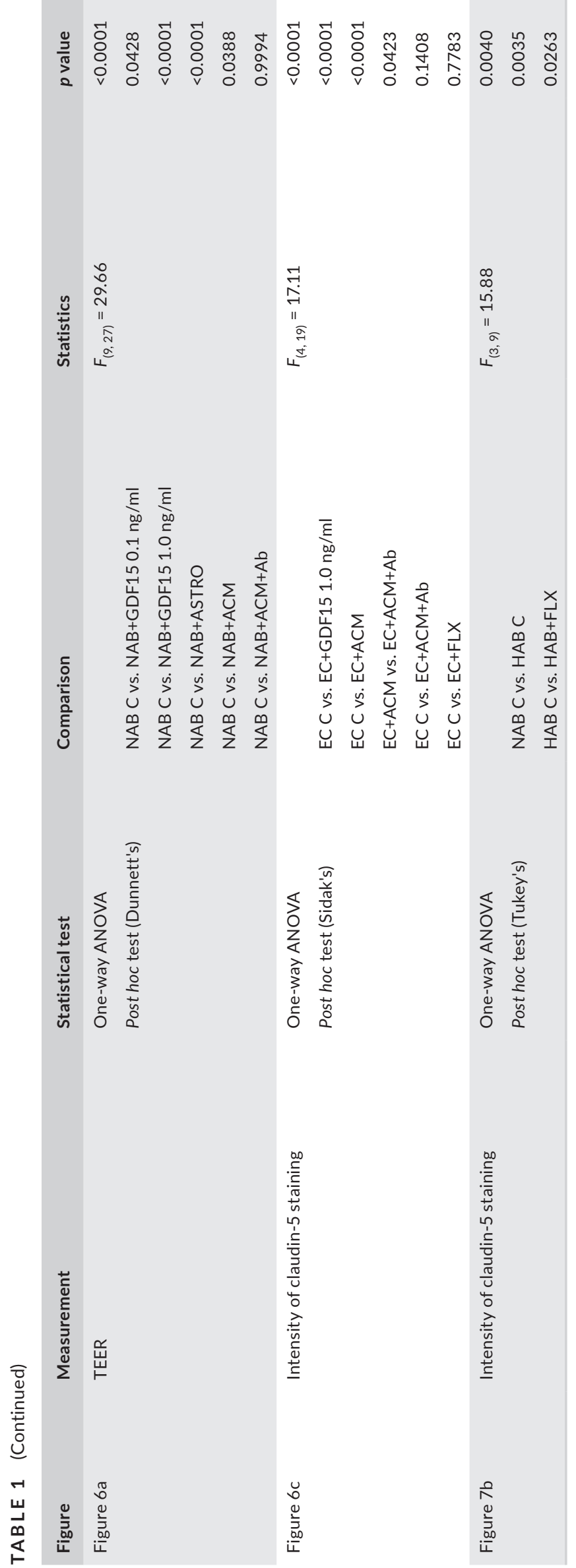

correspond to any general changes in its amount between NAB and $\mathrm{HAB}$ brains (Figure S2, one-way ANOVA, $F_{(3,8)}=1.1571$, $N=3, p=0.2707$ followed by post hoc Fisher's LSD test; Table 1 ). This suggested a generally reduced availability of GDF15 protein in astrocyte processes at the BBB in HAB brains, which might reflect a lack of released factor as well.

Notably, FLX treatment altered the density of co-labeled processes in both $N A B$ and $\mathrm{HAB}$ brains, ultimately rescuing the low values found in HAB brains (Table 1). However, these results were not due to a general modification in total numbers of astrocytes, which were significantly lower in $\mathrm{HAB}$ brains versus NAB brains and did not change upon FLX treatment. Instead, the observed changes were selectively localized to the process compartment (Figure S3, oneway ANOVA, $F_{(3,10)}=5.966, N=3, p=0.0134$ followed by post hoc Fisher's LSD test; Table 1).

\section{3 | GDF15 release increases after FLX administration and soluble GDF15 reshapes and maintains astrocyte processes in physiological conditions}

To determine the physiological impact of GDF15 on healthy cells, we next evaluated morphological changes of NAB primary cortical astrocytes upon FLX treatment and administration of human recombinant GDF15 (hrGDF15, 0.01, 0.1, and $1.0 \mathrm{ng} / \mathrm{ml}$ ). In apparent contrast with in vivo results, we revealed that either treatment doubled the number of total astrocyte processes per cell, which persisted along time (Figure 3a,b, one-way ANOVA, $F_{(3,38)}=9.227$, $N \geq 11, p=0.0005$ followed by post hoc Dunnett's test; Figure 3a,c, two-way ANOVA, factor time $F_{(1,97)}=0.8520, p=0.3583$; factor concentration $F_{(3,97)}=7.954, p \leq 0.0001$; interaction $F_{(3,97)}=1.877$, $N \geq 11, p=0.1386$ followed by post hoc Sidak's test; Table 1 ). Moreover, measurements of GDF15 in ACM upon FLX administration indicated that amounts of released protein were between $\sim 0.5$ and $\sim 1.0 \mathrm{ng} / \mathrm{ml}$, which fell in the range of morphogenic hrGDF15 concentrations (Figure 3d, one-way ANOVA, $F_{(2,24)}=102.8, N=8$, $p=0.0001$ followed by post hoc Dunnett's test; Table 1). To evaluate whether GDF15 alone induced the morphological alterations, we further examined the impact of its downregulation via short interfering RNAs (siRNA) in NAB astrocytes. We thereby confirmed its remodeling role, because the genetic inhibition of gdf15 (knockdown, KD) was sufficient to cause a dramatic reduction in the total number of astrocyte processes per cell (Figure 3e,f, oneway ANOVA, $F_{(3,41)}=3.865, N \geq 10, p=0.0160$ followed by post hoc Dunnett's test; Table 1). Additionally, the investigation of putative signaling pathways downstream of GDF15 showed that the ERK signaling pathway, which is relevant to reshape cellular morphologies through the phosphorylation of ERK1 and ERK2 (ERK1/2; Kumar, Zhang, Swank, Kunz, \& Wu, 2005; Wu, Deisseroth, \& Tsien, 2001), underlined GDF15 effects. These results were confirmed by the pretreatment of cells with U0126, a specific inhibitor of phosphorylated ERK, which completely prevented GDF15-induced 

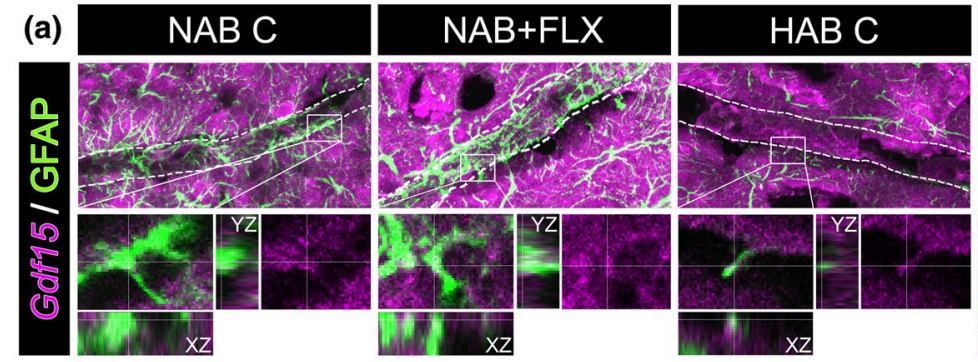

$\mathrm{HAB}+\mathrm{FLX}$

(b)

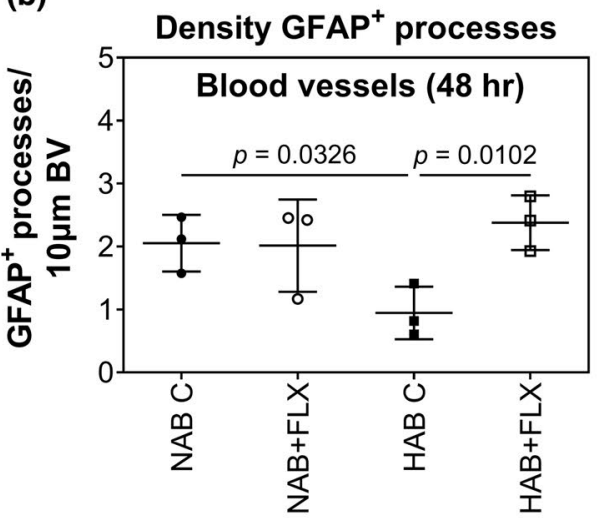

कs (c)
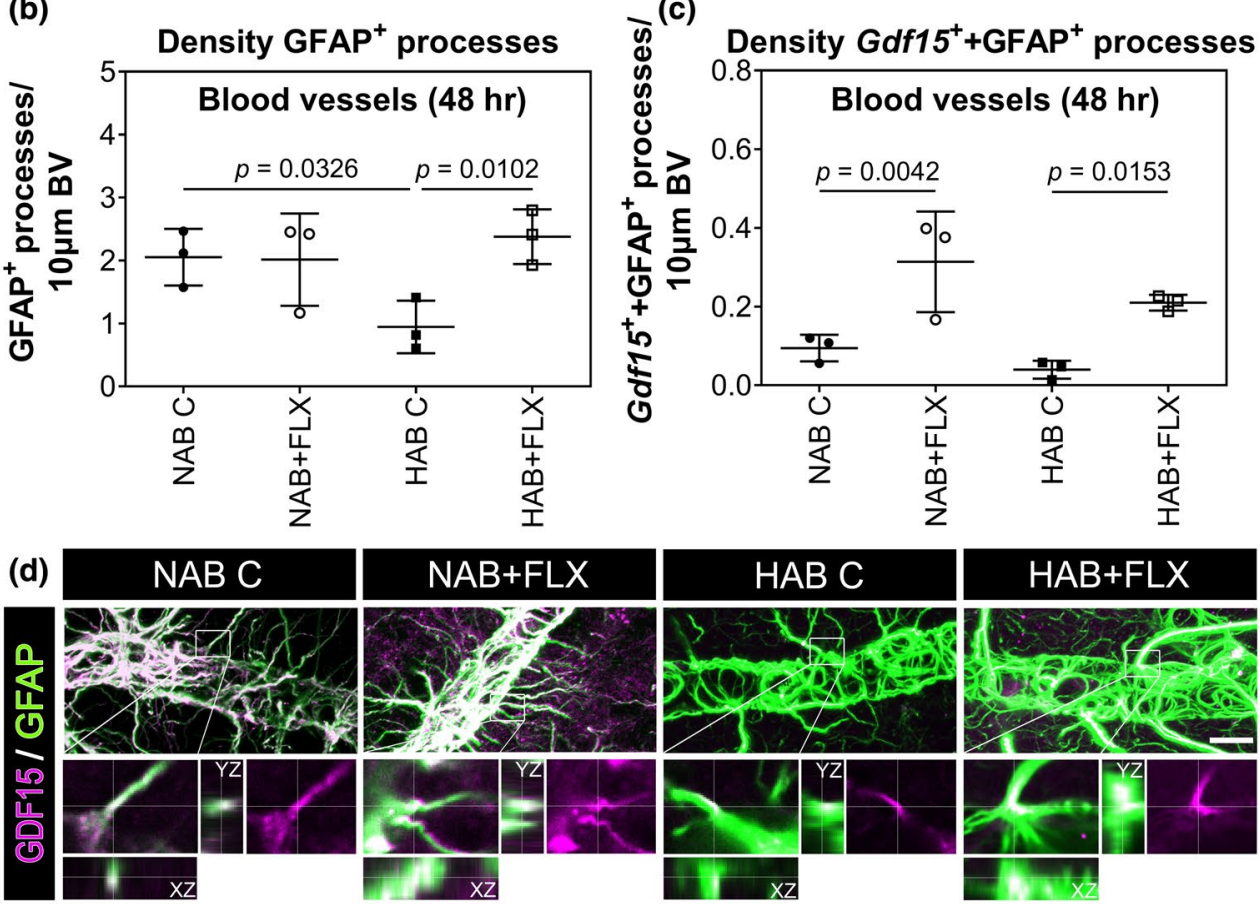

$\mathrm{HAB}+\mathrm{FLX}$

(e)
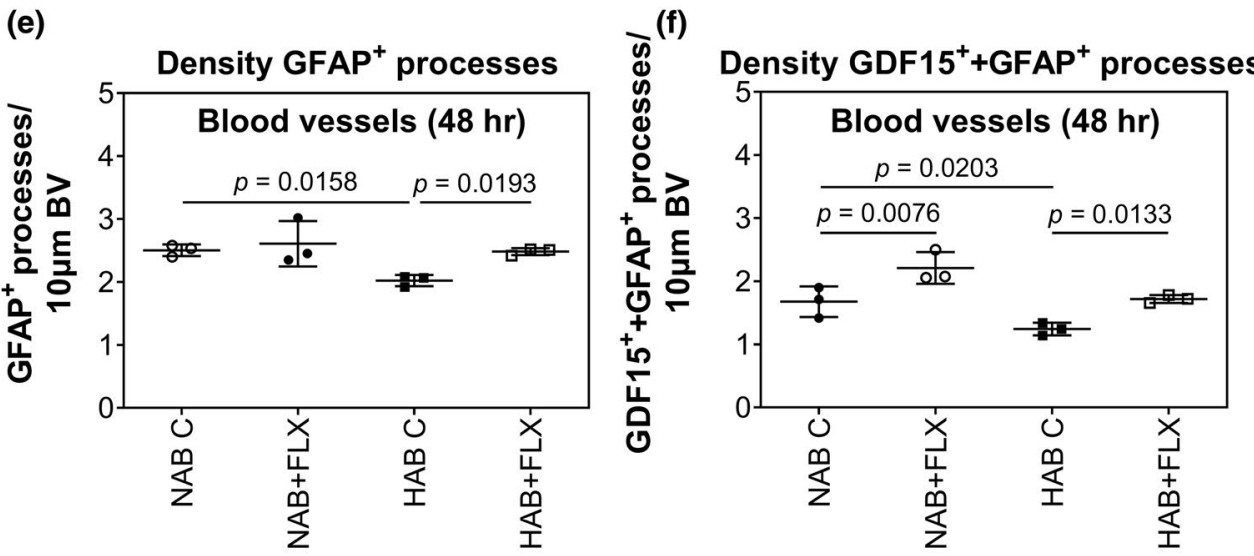

FIGURE 2 HAB animals display a deficiency in GFAP-positive astrocytic processes around BVs of the PFC, which is rescued by a GDF15inducing FLX treatment. (a-d) Representative images delineate GFAP+-labeled astrocyte processes (in green) costained with a probe against gdf15 mRNA (in magenta, a) or an antibody against GDF15 (in magenta, d) around BVs in brains of untreated and FLX-treated NAB and HAB rats ( $N=3$ brains/group). Scale bar $50 \mu \mathrm{m}$ (a), $20 \mu \mathrm{m}$ (d). (b-e) Quantification of differences in density of astrocytic processes (number of GFAP+-processes every $10 \mu \mathrm{m} \mathrm{BV}$ ) around BVs in NAB and HAB control animals before and after FLX treatment. Orthogonal projections showed details of processes which we considered positive for our analysis ( $N=3$ brains/group). C-F Quantification of differences in density of double positive gdf15 $/ \mathrm{GFAP}^{+}$(GDF15 ${ }^{+} / \mathrm{GFAP}^{+}$)-enriched processes in $\mathrm{HAB}$ and NAB animals after FLX treatment (N $=3$ brains/group). Data information: data are presented as mean $\pm S D$; in a,b data are presented as mean $\pm S D$, one-way ANOVA, $p=0.0459$ followed by post hoc Fisher's LSD test; in a,c, one-way ANOVA, $p=0.0048$ followed by post hoc Fisher's LSD test; in d,e, one-way ANOVA, $p=0.0246$ followed by post hoc Fisher's LSD test; in d,f, one-way ANOVA, $p=0.0016$ followed by post hoc Fisher's LSD test. Table 1 for statistic details [Color figure can be viewed at wileyonlinelibrary.com] 
morphological changes. In addition, U0126 alone significantly reduced basal numbers of astrocyte processes in NAB cells to similar extent as gdf15 KD (Figure 3g, H, one-way ANOVA, $F_{(3,92)}=98.95$, $N \geq 22, p=0.0001$ followed by post hoc Tukey's test; Table 1), further supporting the relevance of ERK1/2 as molecular mediator of GDF15 efficacy, but also suggesting its importance to maintain or instruct the basal shape of astrocytes.

\section{4 | GDF15 release increases after}

FLX administration and soluble GDF15

reshapes and maintains astrocyte processes in pathological conditions

Similar results obtained in NAB cells were observed in $\mathrm{HAB}$ cells after treatment with either FLX or hrGDF15, thereby strengthening (a)
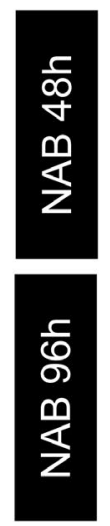

(b)

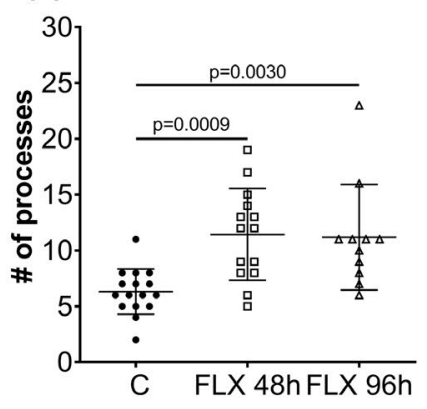

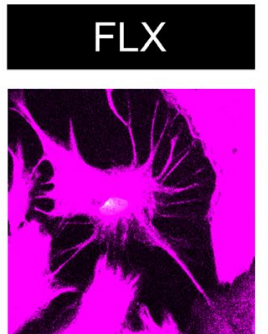

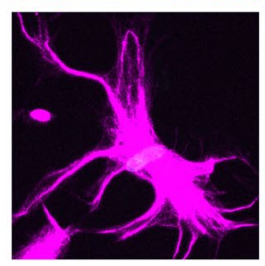

(c)
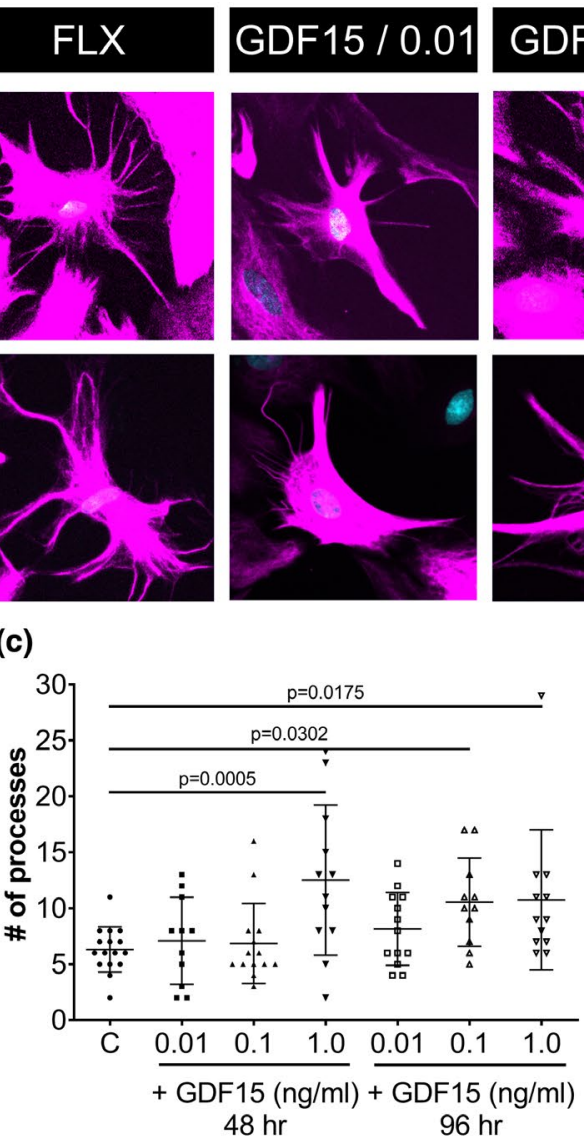

(g)

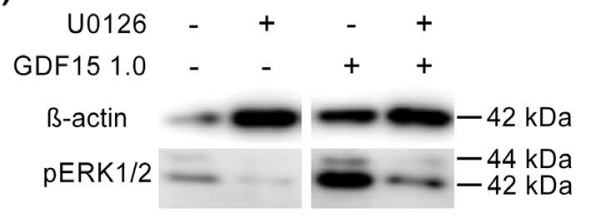

(h)

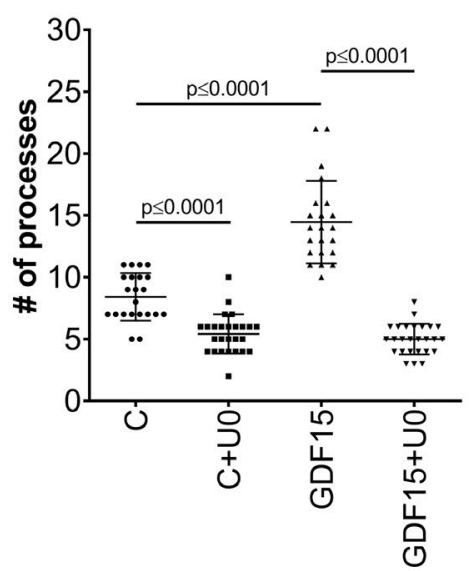

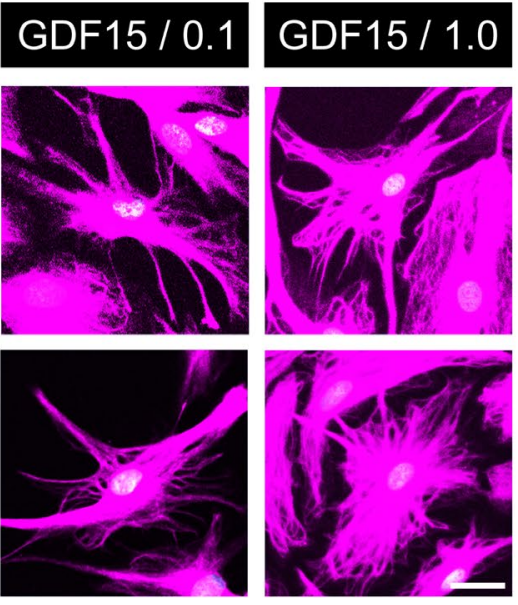

(d)

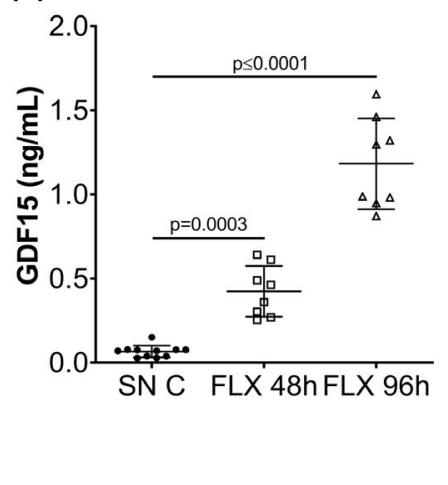

(f)

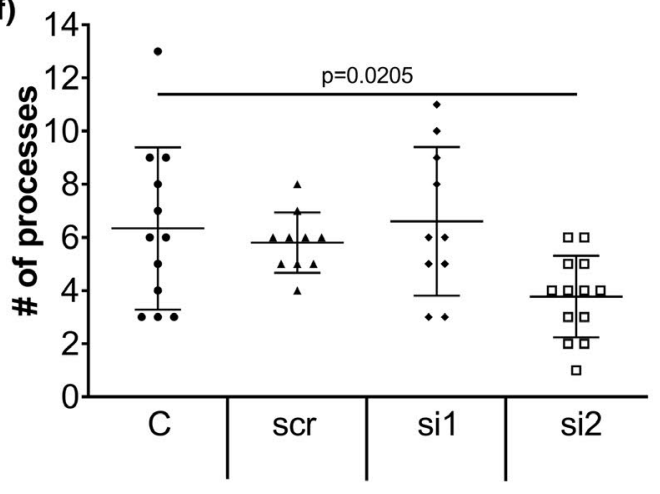

(e)

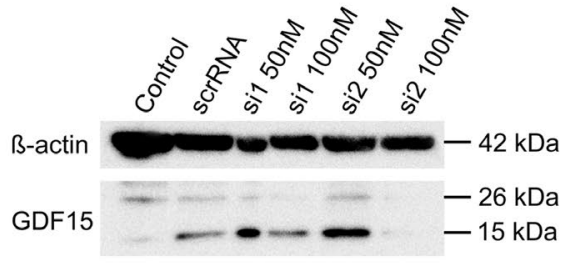


FIGURE 3 GDF15 is necessary to trigger morphological changes in normal astrocytes and it mediates FLX effects. (a) Representative images of NAB primary cortical astrocytes treated for 48 or $96 \mathrm{hr}$ with either FLX $(10 \mu \mathrm{M})$ or with increasing concentrations of hrGDF15 $(0.01,0.1$ and $1 \mathrm{ng} / \mathrm{ml}$ ) and labeled with the astrocyte-specific markers GFAP/S100 $\beta$ (magenta) and DAPI (blue). Scale bar, $10 \mu \mathrm{m}$. (b,c) Analysis of the number of astrocyte processes in NAB treated with either FLX (b) or exogenous hrGDF15 for 48 and 96 hr (c, N $\geq 11$ experiments). (d) ELISA analysis of released GDF15 in media derived from NAB astrocytes after FLX treatment ( $N=8$ experiments). (e,f) siRNA-mediated downregulation of GDF15 in NAB (si1 and si2) shown by Western blot analysis using antibodies directed against GDF15 $(15 / 26 \mathrm{kDa})$ and $\beta$-actin (42 kDa) as a loading control (e) and analysis of the number of processes after GDF15 KD (f, $N \geq 10$ experiments). G,H Primary NAB astrocytes were treated with either U0126 $(20 \mu \mathrm{M})$ or hrGDF15 $(1 \mathrm{ng} / \mathrm{ml})$ alone or in combination (U0126 administered $30 \mathrm{~min}$ prior hrGDF15) for $48 \mathrm{hr}$ and quantification of treatment effects on number of astrocytic processes (h, $N \geq 22$ experiments). Efficacy of treatments on phosphorylated ERK1/2 are shown in a Western blot on primary NAB astrocytes using specific antibodies against pERK1/2 $(44 / 42 \mathrm{kDa})$ and $\beta$-actin (42 kDa) (g). Data information: data are represented as mean $\pm S D$; in a,b, one-way ANOVA, $p=0.0005$ followed by post hoc Dunnett's test; in a,c, two-way ANOVA, concentration effect, $p=0.0001$ followed by post hoc Sidak's test; in 3D, one-way ANOVA, $p \leq 0.0001$ followed by post hoc Dunnett's test; in e,f, one-way ANOVA, $p=0.0160$ followed by post hoc Dunnett's test; in g,h, one-way ANOVA, $p \leq 0.0001$ followed by post hoc Tukey's test. Table 1 for statistic details [Color figure can be viewed at wileyonlinelibrary.com]

our hypothesis about a physiological role of GDF15 as mediator of plasticity-inducing drugs such as FLX and modulator of astrocyte morphology (Figure 4a,b, one-way ANOVA, $F_{(3,33)}=9.901, N \geq 11$, $p=0.0004$ followed by post hoc Dunnett's test; Figure 4a,c, two-way ANOVA: factor time $F(1,84)=0.2035, p=0.6531$; factor concentration $F_{(3,84)}=14.68, p \leq 0.0001$; interaction $F_{(3,84)}=5.240, N \geq 11$, $p=0.0023$ followed by post hoc Sidak's test; Table 1). Even in this case, measurements of released GDF15 in ACM showed its elevated levels upon FLX administration (Figure $4 \mathrm{~d}$, around $0.5 \mathrm{ng} / \mathrm{ml}$, oneway ANOVA, $F_{(2,22)}=10.35, N=8, p=0.0007$ followed by post hoc Dunnett's test; Table 1).

To next determine whether intracellular or secreted GDF15 influenced the morphological remodeling, we treated $\mathrm{HAB}$ astrocytes with $A C M$ derived from $N A B$ astrocytes previously manipulated to stimulate or abrogate GDF15. We showed that both its genetic neutralization as well as the immunological blockade of its secreted form with an anti-GDF15 antibody ("Ab 100/500") caused only moderate changes in basal numbers of astrocyte processes in HAB cells (" $\mathrm{C}$ " vs. "C si2" and "C Ab 500"), probably due to their already lowered levels. However, these treatments completely blocked the morphogenic effects of FLX, thus confirming that astrocyte-derived GDF15 was indeed necessary and sufficient to affect process formation in $\mathrm{NAB}$ as well as in HAB astrocytes (Figure $4 \mathrm{e}$, one-way ANOVA, $F_{(12}$, ${ }_{140)}=8.038, N \geq 10, p=0.0001$ followed by post hoc Fisher's LSD test; Table 1).

\section{5 | GDF15 promotes astrocyte process sprouting}

Recently, it has been shown that GDF10, another member of the TGF $\beta$ superfamily, induces axonal sprouting, thus suggesting a putative role of GDF15 in affecting process sprouting in astrocytes. We therefore subdivided all processes generated after FLX or hrGDF15 treatments in subgroups depending on their differential lengths. This analysis revealed that both treatments preferentially increased the amount of short-sized processes (0-10/10-20 $\mu \mathrm{m}$ ranges) in both $\mathrm{NAB}$ and $\mathrm{HAB}$ astrocytes and at both time points evaluated (48 and $96 \mathrm{hr}$; Figure 5a-f, two-way ANOVA: (a) factor length $F(15,608)=105.4, p \leq 0.0001$; factor treatment $F_{(2,608)}=9.770$, $p \leq 0.0001$; interaction $F_{(30,608)}=4.127, p \leq 0.0001, N \geq 12$, (b) factor length $F_{(15,784)}=63.99, p \leq 0.0001$; factor treatment $F_{(3,784)}=6.060$, $p=0.0004$; interaction $F_{(45,784)}=2.266, p \leq 0.0001, N \geq 12$, (c) factor length $F_{(15,768)}=99.53, p \leq 0.0001$; factor treatment $F_{(3,768)}=4.206$, $p=0.0004$; interaction $F_{(45,768)}=1.681, p=0.0040, N \geq 12$, (d) factor length $F_{(15,768)}=75.00, p \leq 0.0001$; factor treatment $F_{(3,768)}=8.632$, $p \leq 0.0001$; interaction $F_{(45,768)}=2.927, p \leq 0.0001, N \geq 12$, (e) factor length $F_{(15,896)}=80.49, p \leq 0.0001$; factor treatment $F_{(4,896)}=6.297$, $p \leq 0.0001$; interaction $F_{(60,896)}=2.567, p \leq 0.0001, N \geq 12$, (f) factor length $F_{(15,928)}=68.33, p \leq 0.0001$; factor treatment $F_{(4,928)}=9.822$, $p \leq 0.0001$; interaction $F_{(60,928)}=3.175, p \leq 0.0001, N \geq 12$; followed by post hoc Dunnett's test; see Table 1 for statistical details). Our results strongly supported a role of GDF15 in triggering the sprouting of new processes at early time points ( $48 \mathrm{hr}$ ), with additional continuous effects at later time points $(96 \mathrm{hr}$ ). To further explore this hypothesis, we examined $\mathrm{HAB}$ cells exposed to ACM derived from NAB astrocytes previously modified to inhibit or induce GDF15 production. Results revealed that only $H A B$ astrocytes subjected to $A C M$ derived from FLX-treated NAB astrocytes ("HAB+ACM NAB+FLX") showed the expected morphological changes characterized by increased amounts of short-sized processes. On the contrary, ACM from gdf15 KD NAB astrocytes ("HAB+ACM NAB si2+FLX") showed a completely abolished FLX-induced effects (Figure 5g,h, two-way ANOVA, factor length $F_{(15,864)}=81.76, p \leq 0.0001$; factor treatment $F_{(3,864)}=12.92, p \leq 0.0001$; interaction $F_{(45,864)}=5.121, p \leq 0.0001$, $N=12$, followed by post hoc Tukey's test; Table 1 ). These findings supported that secreted GDF15 was indeed acting as a trigger of process sprouting. Moreover, they indicated that GDF15 was the primary effector of FLX-induced morphological remodeling of astrocytes, as its genetic inhibition was sufficient to abrogate the morphogenic effect of ACM derived from FLX-treated NAB astrocytes.

\subsection{GDF15 promotes the strengthening of tight junctions between endothelial cells}

Because of the specific expression of GDF15 around BVs and its peculiar effects on astrocyte processes, which influence BBB properties, we then investigated its functional role for the formation of TJ between ECs. To first examine whether astrocyte-secreted GDF15 influenced barrier functions, we measured TEER of 


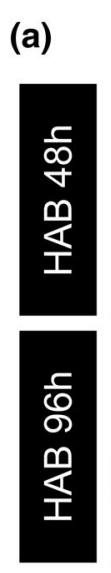

(b)

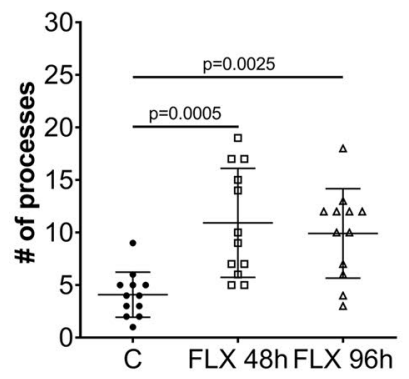

(e)

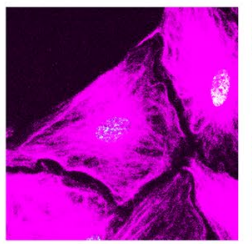

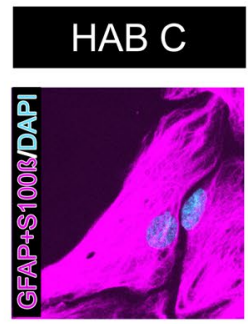

(c)
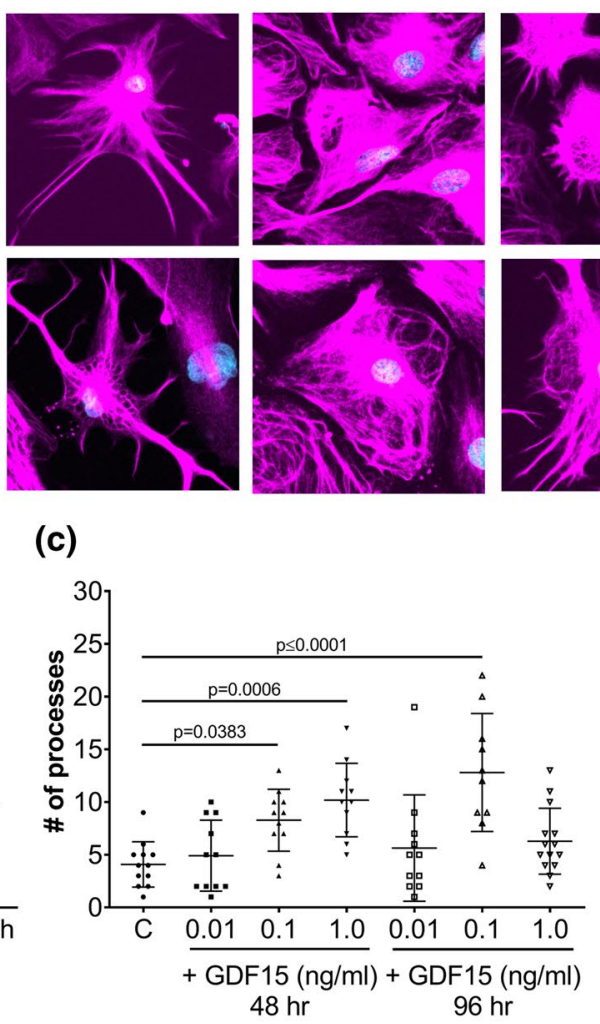

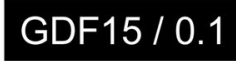

GDF15/1.0

$\mathrm{HAB}+\mathrm{ACM}$
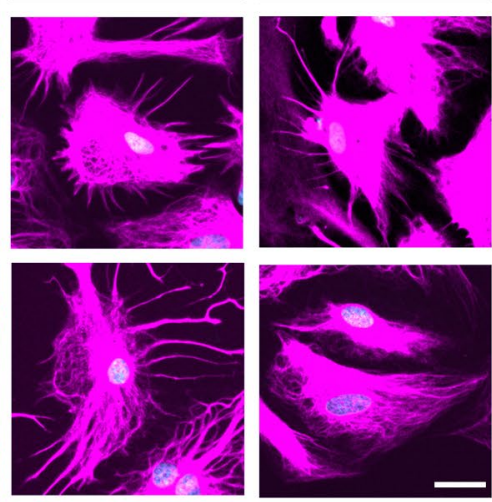

(d)

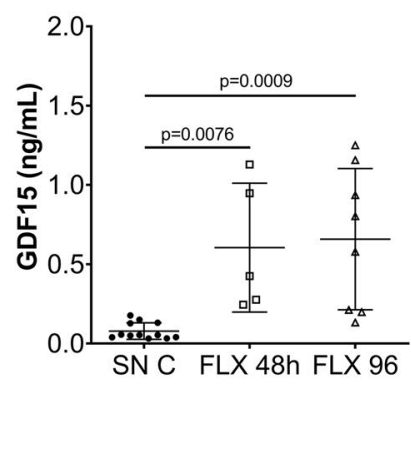

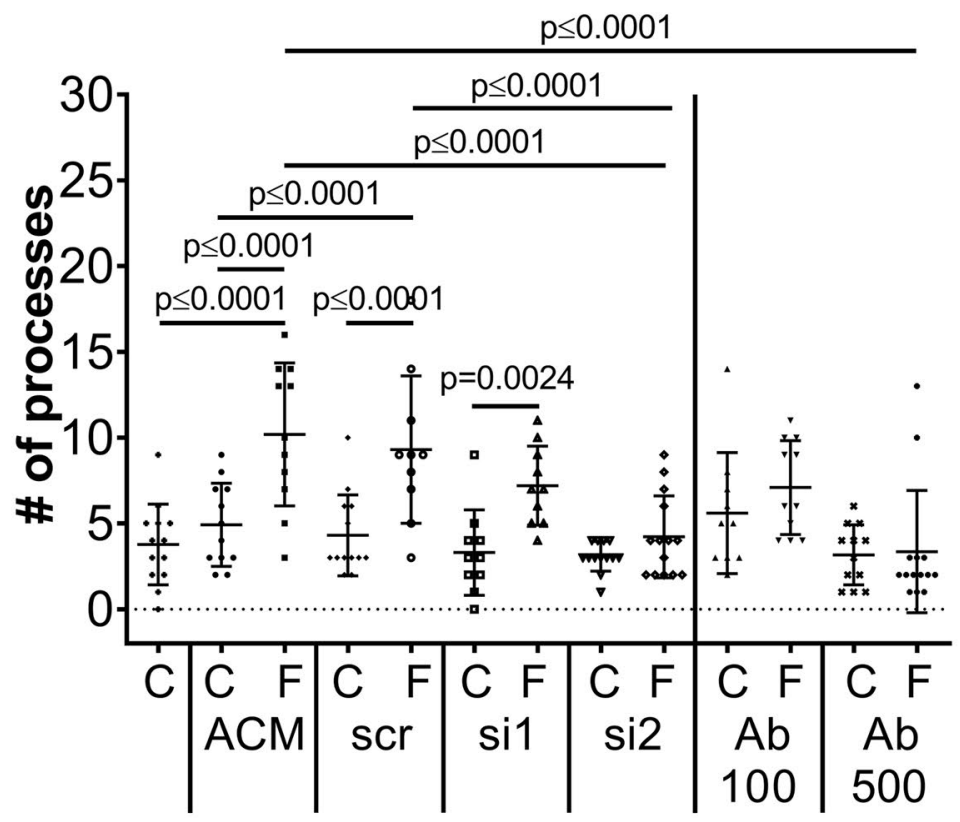

FIGURE 4 GDF15 is sufficient to trigger morphological changes in diseased astrocytes and it mediates FLX effects. (a) Representative images of HAB primary cortical astrocytes treated for 48 or 96 hr with either FLX (10 $\mu \mathrm{M})$ or with increasing concentrations of hrGDF15 (0.01, 0.1 , and $1 \mathrm{ng} / \mathrm{ml}$ ) and labeled with the astrocyte-specific markers GFAP/S100 $\beta$ (magenta) and DAPI (blue). Scale bar, $10 \mu \mathrm{m}$. (b,c) Analysis of the number of astrocyte processes in HAB treated with either FLX (b) or exogenous hrGDF15 for 48 and $96 \mathrm{hr}$ (c, $N \geq 11$ experiments). (d) ELISA analysis of released GDF15 in media derived from HAB astrocytes after FLX treatment ( $N=8$ experiments). (e) GDF15 released from astrocytes is necessary and sufficient to mediate FLX effects on the number of astrocytic processes in HAB cells. HAB cells were treated with ACM from $N A B$ cells that were subjected to pretreatments as indicated ( $N \geq 11$ experiments). Data information: data are represented as mean $\pm S D$; in a,b, one-way ANOVA, $p=0.0004$ followed by post hoc Dunnett's test; in a,c, two-way ANOVA, concentration $\times$ time interaction, $p=0.0023$; concentration effect, $p \leq 0.0001$ followed by post hoc Sidak's test; in d, one-way ANOVA, $p=0.0007$ followed by post hoc Dunnett's test; in e, one-way ANOVA, $p=0.0001$ followed by post hoc Fisher's LSD test. Table 1 for statistic details [Color figure can be viewed at wileyonlinelibrary.com] 
(a)

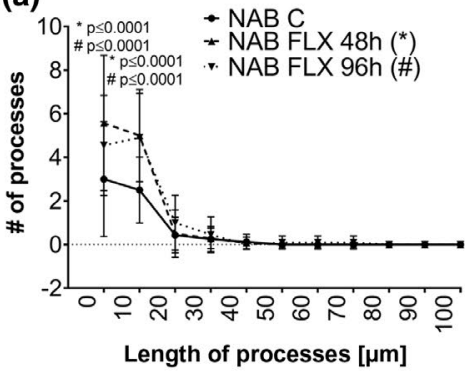

(d)

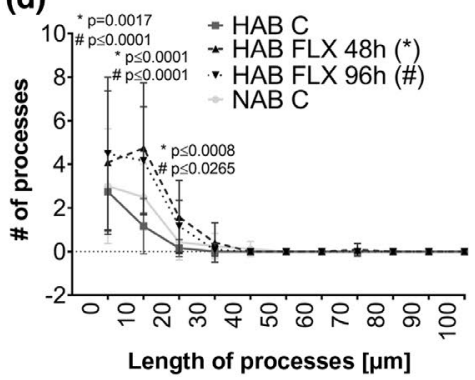

(b)

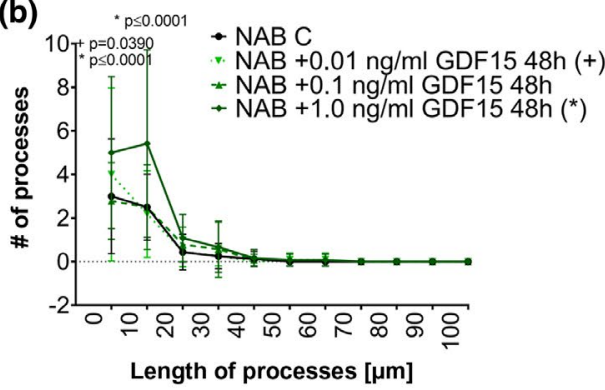

(c)

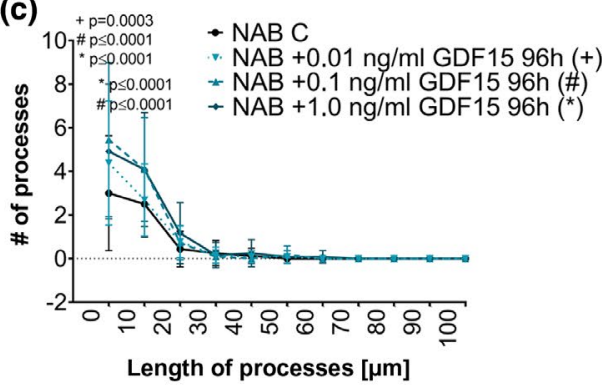

(e)

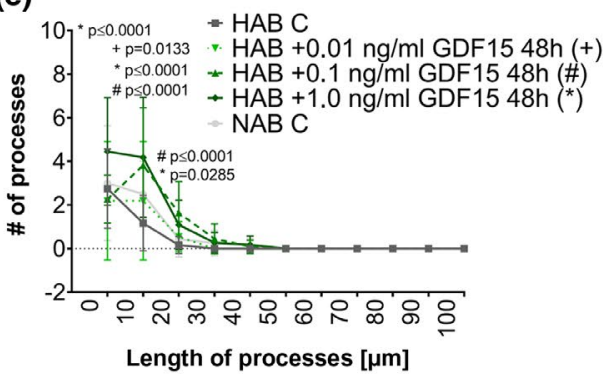

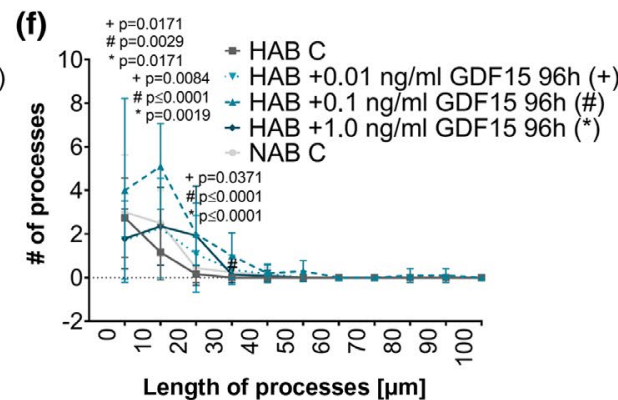

(g)
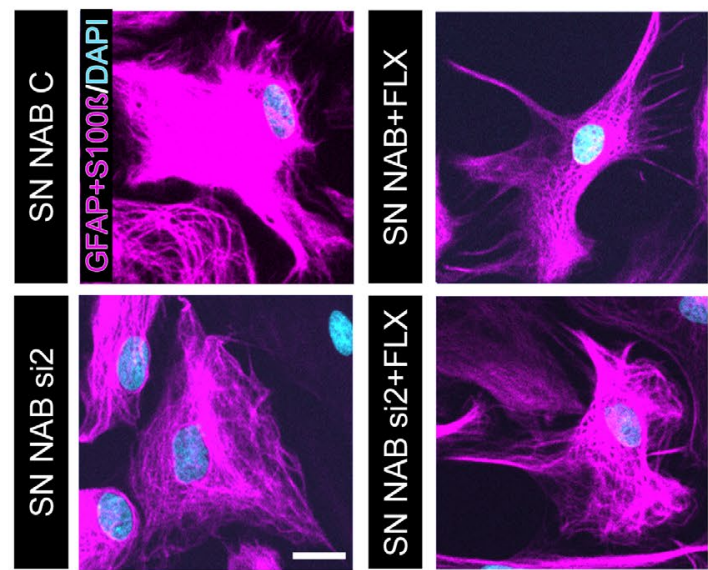

(h) $\quad+p \leq 0.0001$

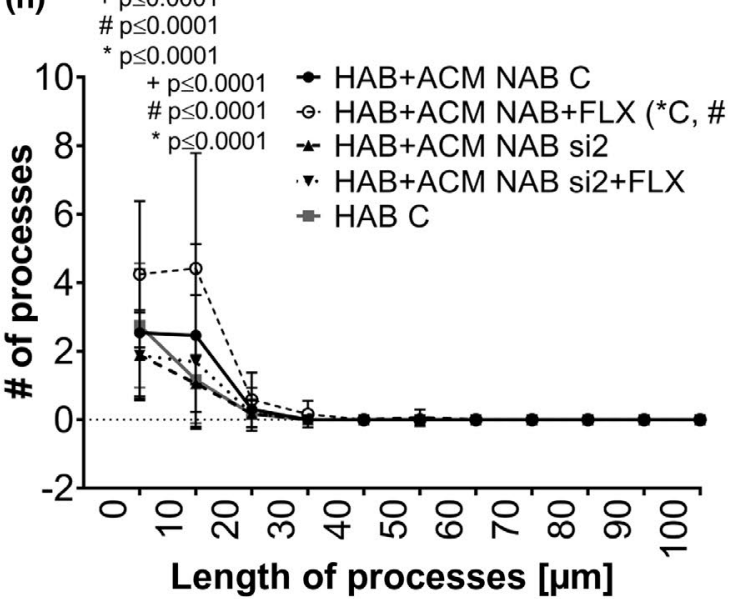

FIGURE 5 GDF15 preferentially promotes sprouting of new astrocyte processes in NAB and HAB cells. (a-f) In depth analysis of the length of astrocytic processes in NAB and HAB astrocytes after administration of either FLX or hrGDF15 (0.01, 0.1, and 1.0 ng/ml) for 48 and $96 \mathrm{hr}(N \geq 12$ experiments). Scale bar, $10 \mu \mathrm{m}$. (g) Representative images of HAB astrocytes (GFAP/S100 $\beta$, magenta) and DAPI (blue) exposed to $A C M$ derived from NAB cells, either without treatment ( $A C M N A B C$ ), with FLX (ACM NAB+FLX), siRNA-mediated KD of GDF15 (ACM NAB si2) or FLX treatment after GDF15 KD (SN NAB si2+FLX) for $48 \mathrm{hr}$. (h) In depth analysis of the number of the lengths of processes in $\mathrm{HAB}$ astrocytes subjected to ACM derived from NAB astrocytes modified as described in $\mathrm{g}$ ( $\mathrm{N} \geq 12$ experiments). Scale bar, $10 \mu \mathrm{m}$. Data information: data are represented as mean $\pm S D$; in a, two-way ANOVA, length $\times$ treatment interaction, $p \leq 0.0001$; length effect, $p \leq 0.0001$; treatment effect, $p \leq 0.0001$ followed by post hoc Dunnett's test; in $\mathrm{b}$, two-way ANOVA, length $\mathrm{x}$ treatment interaction, $p \leq 0.0001$; length effect, $p \leq 0.0001$; treatment effect, $p=0.0004$ followed by post hoc Dunnett's test; in c, two-way ANOVA, length $\times$ treatment interaction, $p=0.0040$; length effect, $p=0.0001$; treatment effect, $p=0.0058$ followed by post hoc Dunnett's test; in $\mathrm{d}$, two-way ANOVA, length $\times$ treatment interaction, $p \leq 0.0001$; length effect, $p \leq 0.0001$; treatment effect, $p \leq 0.0001$ followed by post hoc Dunnett's test; in e, two-way ANOVA, length $\times$ treatment interaction, $p=0.0001$; length effect, $p=0.0001$; treatment effect, $p=0.0001$ followed by post hoc Dunnett's test; in $\mathrm{f}$, two-way ANOVA, length $\times$ treatment interaction, $p=0.0001$; length effect, $p=0.0001$; treatment effect, $p \leq 0.0001$ followed by post hoc Dunnett's test; in $\mathrm{G}, \mathrm{H}$, two-way ANOVA, length $\times$ treatment interaction, $p \leq 0.0001$; length effect, $p \leq 0.0001$; treatment effect, $p \leq 0.0001$ followed by post hoc Tukey's test. Table 1 for statistic details [Color figure can be viewed at wileyonlinelibrary.com]

ECs grown in different conditions. We revealed that ECs treated with soluble hrGDF15 as well as cocultured in the presence of intact astrocytes or exposed to ACM derived from NAB astrocytes showed higher TEER values than control cells, whereas blockade of soluble GDF15 in ACM was sufficient to completely abolish this effect. Moreover, these effects were triggered by GDF15 but not by other substances known to be released by astrocytes, such as NGF or TGFß (Figure 6a, one-way ANOVA, $F_{(9,27)}=29.66$, 
(a)
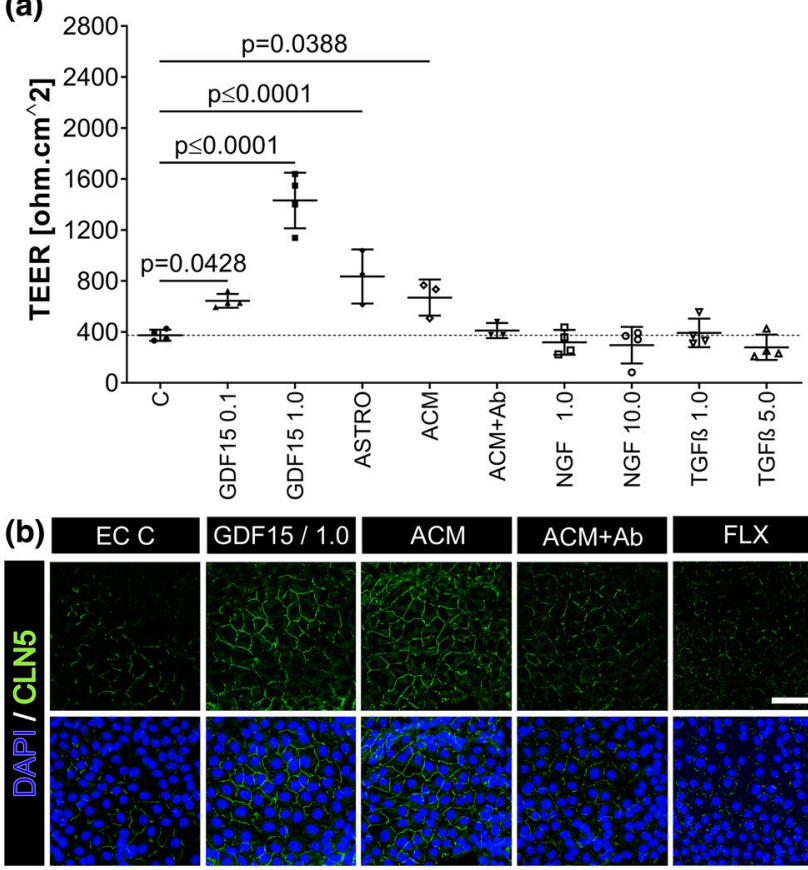

ACM

$\mathrm{ACM}+\mathrm{Ab}$

FLX

(c)

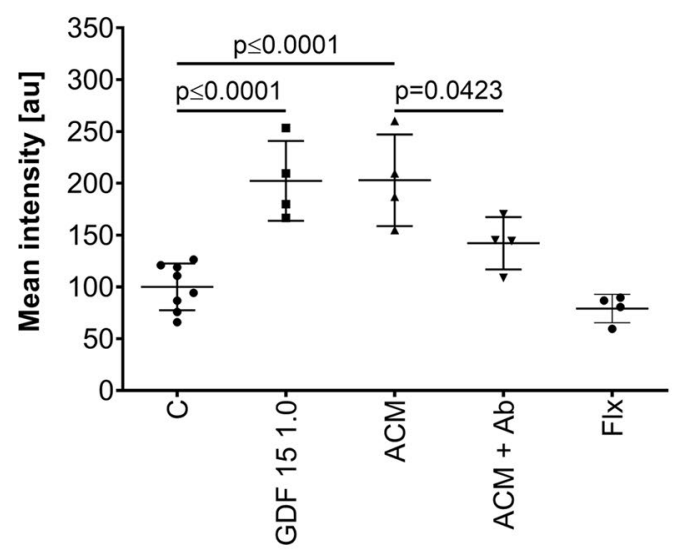

FIGURE 6 GDF15 favors increased barrier properties and the strengthening of tight junctions in EC. (a) Analysis of transendothelial electrical resistance (TEER) in ECs upon different treatment conditions. (b) Representative images of ECs labeled with an antibody against claudin-5, a marker of tight junctions (CLN5, green) and DAPI (blue) exposed to GDF15, ACM, and $A C M+A b$ (anti-GDF15). Scale bar, $50 \mu \mathrm{m}$. (c) Quantification of CLN5 intensity between $\mathrm{ECs}$ after the respective treatments $(N \geq 12$ experiments). Data information: data are represented as mean $\pm S D$; in a, one-way ANOVA, $p \leq 0.0001$ followed by post hoc Dunnett's test; c, one-way ANOVA, $p \leq 0.0001$ followed by post hoc Sidak's test. Table 1 for statistic details [Color figure can be viewed at wileyonlinelibrary.com]

$N=4, p \leq 0.0001$ followed by post hoc Dunnett's test; Table 1). Remarkably, the effect of hrGDF15 and ACM on TEER specifically corresponded to a selective GDF15-dependent increased claudin-5 (CLN5) expression around EC, with no further changes mediated by treatment with FLX alone, further strengthening our hypothesis on a physiological role of GDF15 on BBB properties (Figure 6b,c, one-way ANOVA, $F_{(4,19)}=17.11, N=3-4, p \leq 0.0001$ followed by post hoc Sidak's test; Table 1).
To evaluate whether deficits in PAP in HAB animals might correspond to an impaired CLN5 expression in ECs, we next used IHC to compare CLN5 expression between $\mathrm{NAB}$ and $\mathrm{HAB}$ brains ex vivo before and after treatment with FLX, our GDF15-inducing drug. This comparison revealed a significant reduction of CLN5 expression in HAB ECs along with the impaired astrocyte process complexity. Additionally, both CLN5 and astrocyte morphological deficits were fully and simultaneously rescued by FLX administration, further supporting the putative impact of locally released GDF15 on BBB properties, beyond its effects on astrocyte processes (Figure 7a,b, one-way ANOVA, $F_{(3,9)}=15.88, N=3, p=0.0040$ followed by post hoc Tukey's test; Table 1).

\section{4 | DISCUSSION}

Our data show that GDF15 expression is increased in primary cortical astrocytes and PAP upon treatment with FLX, a drug which affects astrocyte plasticity (Di Benedetto et al., 2016). We also provide evidence that hrGDF15 alone triggers astrocyte morphological changes apposed to those observed after selective drug treatments. Moreover, our data indicate that secreted GDF15 is necessary for these effects and its genetic or immunological neutralizations could not be compensated by any other astrocytederived released factors. Our results highlight a new physiological and cell autonomous role of astrocyte-derived GDF15 on the reshaping of astrocytes' morphology which may complement its noncell autonomous effects on neurons (Strelau et al., 2009), thereby broadening the spectrum of cellular targets for GDF15 trophic functions. Several astrocyte-dependent effectors have been identified which play functional roles at synaptic locations (Chung et al., 2013; Kucukdereli et al., 2011), but not much is known about astrocyte-derived molecular triggers of vascular dynamic remodeling processes which might be relevant to regulate formation and/or functions of the BBB. Besides the mediation of transport functions at the BBB and regulation of blood flow (Cabezas et al., 2014), astrocyte processes around BVs are essential to influence BBB permeability through the induction of junctional proteins such as claudins or occludins. Few studies have evidenced the role of members of the fibroblast growth factor (FGF) family in affecting BBB properties (Reuss et al., 2003), although more recent work has questioned the necessity of such factors, and of astrocytes in general, for the development of the BBB (Saunders et al., 2016). Moreover, although FGF signaling has also been shown to induce similar morphological changes in astrocytes to the ones we observed (Goldshmit et al., 2014; Kalman, Gomperts, Hardy, Kitamura, \& Bishop, 1999; Kang, Lee, Han, Choi, \& Song, 2014), it may provoke reactive astrogliosis, which is considered detrimental for surrounding cells. Thus, an excessive FGF signaling activity might in fact compromise BBB permeability and favor an uncontrolled exchange of blood-borne molecules (Sofroniew \& Vinters, 2010). Previous work has also revealed the essential role of astrocytic Sonic Hedgehog (Shh) signaling to enhance BBB integrity 

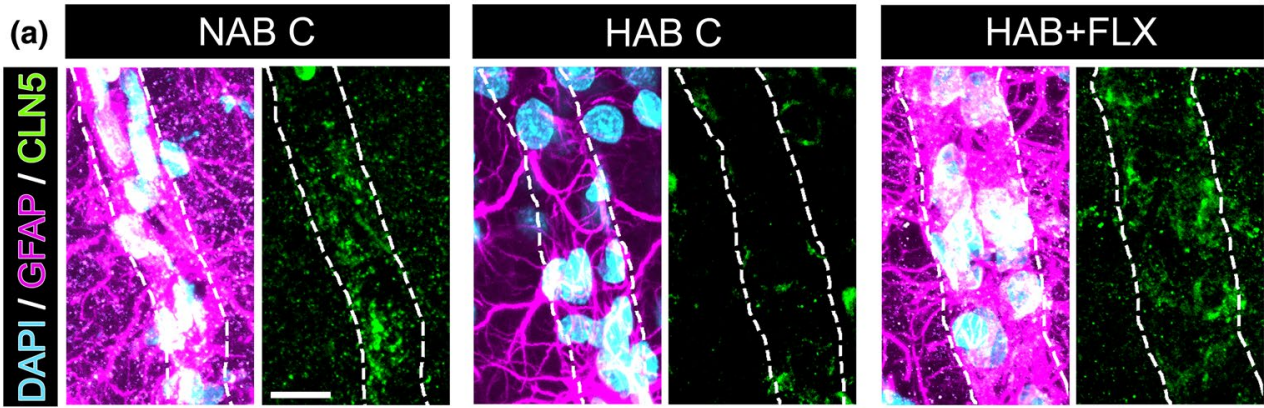

(b)

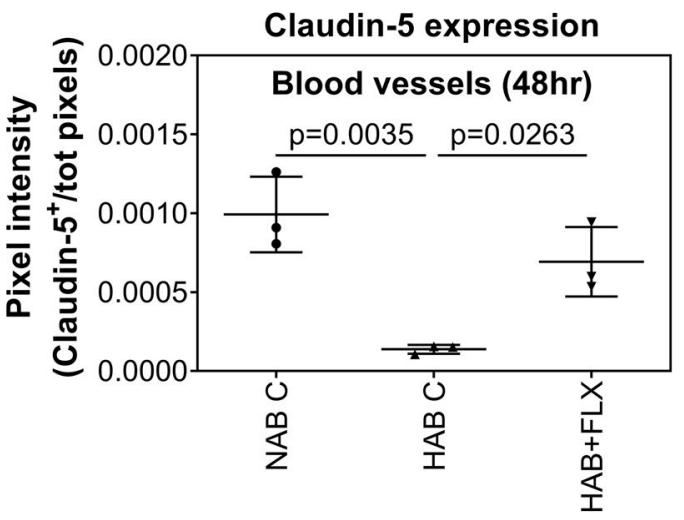

FIGURE 7 GDF15-inducing drug FLX promotes the rescue of CLN5 deficits in HAB EC. (a) Representative images of ECs in NAB and HAB brains labeled with antibodies against GFAP/S100ß (magenta), claudin-5 (CLN5, green), and DAPI (blue) exposed to FLX treatment. Scale bar, $20 \mu \mathrm{m}$. (b) Quantification of CLN5 intensity between ECs after the respective treatments ( $N=3$ brains/group). Data information: data are represented as mean $\pm S D$; in b, one-way ANOVA, $p=0.0040$ followed by post hoc Tukey's test. Table 1 for statistic details [Color figure can be viewed at wileyonlinelibrary.com]

and rescue its deficits in animal models of disease (Alvarez et al., 2011). However, these studies did not detect any simultaneous roles of astrocyte-secreted proteins on astrocytes and ECs. Such bidirectional effects might be relevant in diseases, which display concurrent impaired astrocyte morphology/function and altered BBB properties (Cabezas et al., 2014). With respect to molecular mechanisms beyond these remodeling effects, we identified the ERK signaling pathway as a driver of the observed modifications in astrocytes upon GDF15 stimulation (Figure 2). Considering previous studies showing that the ERK pathway is affected in depressive disorder (MDD; Duric et al., 2010) and astrocytes are morphologically impaired in both human postmortem brain tissues of depressed patients and in HAB brains (Barbara Di Benedetto et al., 2016; Rajkowska \& Stockmeier, 2013), our results on reduced astrocyte processes upon ERK inhibition corroborate the hypothesis that deficits in astrocyte complexity may underlie the pathogenesis of MDD.

The in vitro experiments (Figure 3) revealed an apparent contrast with results obtained ex vivo in NAB brains after FLX treatment, because cells in vitro responded to FLX with an increased number of processes, which did not appear ex vivo. However, in vitro we could not distinguish perivascular and non-perivascular processes, making this approach very informative about general drug effects, but not completely equivalent to ex vivo results. A possible explanation for this apparent inconsistency between in vitro and ex vivo results might be that we underestimated FLX effects ex vivo because of the preferential increase in short-sized processes (Figure 5) raised upon FLX treatment, which may have fallen beyond detectability levels in IHC. An alternative reason for such differences may rely on putative clearance effects of adjacent cells on released GDF15 in the extracellular space, which might have hampered its availability in NAB brains to exert modulatory effects on astrocyte processes in physiological conditions.

Using HAB rats, an animal model of anxiety- and depression-like behavior which presents a disrupted astrocyte process complexity around BVs (Di Benedetto et al., 2016), we identified a physiological role of GDF15 on BV-associated astrocytes and on junctional proteins, strengthening the link between an increased gdf15/GDF15 expression in astrocyte processes at BBB locations with functional effects on barrier sealing properties. Indeed, hrGDF15 specifically enhanced TEER values in ECs in vitro to levels comparable to both ECs co-cultured with astrocytes and exposed to ACM (Figure 6). Moreover, the reduced direct apposition of astrocyte processes on ECs in HAB brains corresponded to compromised CLN5 expression (Figure 7a,b). Further work is necessary to examine how gdf15 expression is regulated during development, particularly in the early postnatal period when astrocytes and EC precursors start interacting to modulate the maturation of the neurovascular unit (Obermeier et al., 2013) or during aging, when changes in expression of ECspecific transporter proteins may influence BBB functions. 
Further functions of astrocytes might be carried out by their processes, which simultaneously contact synaptic and vascular compartments, thereby forming a functional unit that senses the "brain state" and consequently secrete factors in the bloodstream as a reflection of this state. Some papers have already reported proteomic analysis of blood samples from mood disorder patients which evidenced altered concentrations of glia-specific proteins, suggesting a glia cell pathology (Schroeter, Sacher, Steiner, Schoenknecht, \& Mueller, 2013). A limitation in our study is surely represented by the examination of drug effects and pathological signatures of disease only in male rats. Further studies are currently ongoing to compare whether differences in astrocyte processes and/or drug effects may occur in male and female rats. However, our findings are of additional biomedical relevance as they open the venue to screen for selective diagnostic markers of a specific cellular brain pathology. Such studies may be the first step to initiate translational approaches aimed at identifying glial-specific treatment options.

\section{ACKNOWLEDGMENTS}

The authors thank Björn Schwalb and Achim Thresch (LMU Munich) for supporting with the GO analysis of the microarray's results; Nahid Yusufi for helping with the validation of microarray's results in astrocytes (MPI of Psychiatry); Eva M Wagner, Peter Weber, and Claudia Kühne for technical assistance with the establishment of ISH experiments and the execution of the microarray screening (MPI of Psychiatry). We additionally thank: Erwin van den Burg, Rodrigue Maloumby, Andrea Havasi and Doris Bayerl (University of Regensburg) for helping with breeding and injections/perfusions of animals for the ex vivo experiments; Salina Begum, Petra Störtebecker, and Heike Hallof-Büstrich (University of Regensburg) for helping with tissue stainings and Martina Domani (University of Regensburg) for supporting with cell culture.

\section{COMPETING INTEREST}

The authors declare no competing financial interests.

\section{DATA AVAILABILITY STATEMENT}

Data discussed in this publication are deposited in NCBI's Gene Expression Omnibus and are accessible through GEO Series accession number GSE89873 (https://www.ncbi.nlm.nih.gov/geo/query/ acc.cgi?acc=GSE89873).

\section{AUTHOR CONTRIBUTIONS}

Conceptualization, B.D.B.; Methodology, V.A.M., F.Z., S.R., J.K., B.P., S.U. and B.D.B.; Investigation, V.A.M., F.Z., S.R., J.K., S.U. and B.D.B.; Writing -Original Draft, V.A.M. and B.D.B.; Writing -Review \& Editing, V.A.M., F.Z. and B.D.B.; Funding Acquisition, B.D.B., R.R. and I.D.N.; Resources, B.P. and J.M.D. and I.D.N.; Supervision, B.D.B.; Project administration, B.D.B.

\section{DECLARATION OF TRANSPARENCY}

The authors, reviewers, and editors affirm that in accordance with the policies set by the Journal of Neuroscience Research, this manuscript presents an accurate and transparent account of the study being reported and that all critical details describing the methods and results are present.

ORCID

Barbara Di Benedetto iD https://orcid.org/0000-0001-6537-9072

\section{REFERENCES}

Alvarez, J. I., Dodelet-Devillers, A., Kebir, H., Ifergan, I., Fabre, P. J., Terouz, S., ... Prat, A. (2011). The Hedgehog pathway promotes blood-brain barrier integrity and CNS immune quiescence. Science, 334(6063), 1727-1731. https://doi.org/10.1126/science.1206936

Alvarez, J. I., Katayama, T., \& Prat, A. (2013). Glial influence on the blood brain barrier. Glia, 61(12), 1939-1958. https://doi.org/10.1002/ glia. 22575

Beiderbeck, D. I., Reber, S. O., Havasi, A., Bredewold, R., Veenema, A. H., \& Neumann, I. D. (2012). High and abnormal forms of aggression in rats with extremes in trait anxiety-Involvement of the dopamine system in the nucleus accumbens. Psychoneuroendocrinology, 37(12), 1969-1980. https://doi.org/10.1016/j.psyneuen.2012.04.011

Boulay, A.-C., Saubaméa, B., Adam, N., Chasseigneaux, S., Mazaré, N., Gilbert, A., ... Cohen-Salmon, M. (2017). Translation in astrocyte distal processes sets molecular heterogeneity at the gliovascular interface. Cell Discovery, 3, 17005. https://doi.org/10.1038/celld isc. 2017.5

Boyan, B. D., Hyzy, S. L., Pan, Q., Scott, K. M., Coutts, R. D., Healey, R., \& Schwartz, Z. (2016). 24R,25-Dihydroxyvitamin D3 protects against articular cartilage damage following anterior cruciate ligament transection in male rats. PLoS ONE, 11(8), e0161782. https:// doi.org/10.1371/journal.pone.0161782

Cabezas, R., Avila, M., Gonzalez, J., El-Bacha, R. S., Baez, E., GarciaSegura, L. M., ... Barreto, G. E. (2014). Astrocytic modulation of blood brain barrier: Perspectives on Parkinson's disease. Frontiers in Cellular Neuroscience, 8, 211. https://doi.org/10.3389/fncel.2014.00211

Chen, S., Einspanier, R., \& Schoen, J. (2015). Transepithelial electrical resistance (TEER): A functional parameter to monitor the quality of oviduct epithelial cells cultured on filter supports. Histochemistry and Cell Biology, 144(5), 509-515. https://doi.org/10.1007/s0041 8-015-1351-1

Chung, W.-S., Clarke, L. E., Wang, G. X., Stafford, B. K., Sher, A., Chakraborty, C., ... Barres, B. A. (2013). Astrocytes mediate synapse elimination through MEGF10 and MERTK pathways. Nature, 504(7480), 394-400. https://doi.org/10.1038/nature12776

Di Benedetto, B., Malik, V. A., Begum, S., Jablonowski, L., GómezGonzález, G. B., Neumann, I. D., \& Rupprecht, R. (2016). Fluoxetine requires the endfeet protein aquaporin- 4 to enhance plasticity of astrocyte processes. Frontiers in Cellular Neuroscience, 10(8), 62-73. https://doi.org/10.3389/fncel.2016.00008

Di Benedetto, B., \& Rupprecht, R. (2013). Targeting glia cells: Novel perspectives for the treatment of neuropsychiatric diseases. Current Neuropharmacology, 11(2), 171-185. https://doi.org/10.2174/15701 $59 \times 11311020004$

Dunning, M. J., Smith, M. L., Ritchie, M. E., \& Tavare, S. (2007). beadarray: R classes and methods for Illumina bead-based data. Bioinformatics, 23(16), 2183-2184. https://doi.org/10.1093/bioin formatics/btm311

Duric, V., Banasr, M., Licznerski, P., Schmidt, H. D., Stockmeier, C. A., Simen, A. A., ... Duman, R. S. (2010). A negative regulator of MAP 
kinase causes depressive behavior. Nature Medicine, 16(11), 13281332. https://doi.org/10.1038/nm.2219

Goldshmit, Y., Frisca, F., Pinto, A. R., Pébay, A., Tang, J.-K., Siegel, A. L., ... Currie, P. D. (2014). Fgf2 improves functional recovery-decreasing gliosis and increasing radial glia and neural progenitor cells after spinal cord injury. Brain and Behavior, 4(2), 187-200. https://doi. org/10.1002/brb3.172

Hu, B.-L., Shi, C., Lei, R.-E., Lu, D.-H., Luo, W., Qin, S.-Y., ... Jiang, H.-X. (2016). Interleukin-22 ameliorates liver fibrosis through miR-200a/ beta-catenin. Scientific Reports, 6, 36436. https://doi.org/10.1038/ srep36436

Hu, Y., Lao, L., Mao, J., Jin, W., Luo, H., Charpentier, T., ... Wu, J. (2017). Armc5 deletion causes developmental defects and compromises T-cell immune responses. Nature Communications, 8, 13834. https:// doi.org/10.1038/ncomms13834

Huber, W., von Heydebreck, A., Sultmann, H., Poustka, A., \& Vingron, M. (2002). Variance stabilization applied to microarray data calibration and to the quantification of differential expression. Bioinformatics, 18(Suppl. 1), S96-S104.

Kalman, D., Gomperts, S. N., Hardy, S., Kitamura, M., \& Bishop, J. M. (1999). Ras family GTPases control growth of astrocyte processes. Molecular Biology of the Cell, 10(5), 1665-1683.

Kang, K., Lee, S. W., Han, J. E., Choi, J. W., \& Song, M. R. (2014). The complex morphology of reactive astrocytes controlled by fibroblast growth factor signaling. Glia, 62(8), 1328-1344. https://doi. org/10.1002/glia.22684

Kucukdereli, H., Allen, N. J., Lee, A. T., Feng, A., Ozlu, M. I., Conatser, L. M., ... Eroglu, C. (2011). Control of excitatory CNS synaptogenesis by astrocyte-secreted proteins Hevin and SPARC. Proceedings of the National Academy of Sciences of the United States of America, 108(32), E440-E449. https://doi.org/10.1073/pnas.1104977108

Kumar, V., Zhang, M. X., Swank, M. W., Kunz, J., \& Wu, G. Y. (2005). Regulation of dendritic morphogenesis by Ras-PI3K-Akt-mTOR and Ras-MAPK signaling pathways. Journal of Neuroscience, 25(49), 11288-11299. https://doi.org/10.1523/JNEUROSCI.2284-05.2005

Lee, K. M., \& MacLean, A. G. (2015). New advances on glial activation in health and disease. World Journal of Virology, 4(2), 42-55. https://doi. org/10.5501/wjv.v4.i2.42

Li, S., Nie, E. H., Yin, Y., Benowitz, L. I., Tung, S., Vinters, H. V., ... Carmichael, S. T. (2015). GDF10 is a signal for axonal sprouting and functional recovery after stroke. Nature Neuroscience, 18(12), 17371745. https://doi.org/10.1038/nn.4146

Mazare, N., Gilbert, A., Boulay, A. C., Rouach, N., \& Cohen-Salmon, M. (2018). Connexin 30 is expressed in a subtype of mouse brain pericytes. Brain Structure and Function, 223(2), 1017-1024. https://doi. org/10.1007/s00429-017-1562-4

Menard, C., Pfau, M. L., Hodes, G. E., Kana, V., Wang, V. X., Bouchard, S., ... Russo, S. J. (2017). Social stress induces neurovascular pathology promoting depression. Nature Neuroscience, 20(12), 1752-1760. https://doi.org/10.1038/s41593-017-0010-3

Obermeier, B., Daneman, R., \& Ransohoff, R. M. (2013). Development, maintenance and disruption of the blood-brain barrier. Nature Medicine, 19, 1584-1596. https://doi.org/10.1038/nm.3407

Omidi, Y., \& Barar, J. (2012). Impacts of blood-brain barrier in drug delivery and targeting of brain tumors. Bioimpacts, 2(1), 5-22. https://doi. org/10.5681/bi.2012.002

Pekny, M., Pekna, M., Messing, A., Steinhäuser, C., Lee, J.-M., Parpura, V., ... Verkhratsky, A. (2016). Astrocytes: A central element in neurological diseases. Acta Neuropathologica, 131(3), 323-345. https://doi. org/10.1007/s00401-015-1513-1

Pfaffl, M. W. (2001). A new mathematical model for relative quantification in real-time RT-PCR. Nucleic Acids Research, 29(9), e45.

$R$ Core Team. (2015). R: A language and environment for statistical computing. R Foundation for Statistical Computing. Retrieved from https:// www.R-project.org/
Rajkowska, G., Hughes, J., Stockmeier, C. A., Javier Miguel-Hidalgo, J., \& Maciag, D. (2013). Coverage of blood vessels by astrocytic endfeet is reduced in major depressive disorder. Biological Psychiatry, 73(7), 613-621. https://doi.org/10.1016/j.biopsych.2012.09.024

Rajkowska, G., \& Stockmeier, C. A. (2013). Astrocyte pathology in major depressive disorder: Insights from human postmortem brain tissue. Current Drug Targets, 14(11), 1225-1236.

Reuss, B., Dono, R., \& Unsicker, K. (2003). Functions of fibroblast growth factor (FGF)-2 and FGF-5 in astroglial differentiation and bloodbrain barrier permeability: Evidence from mouse mutants. Journal of Neuroscience, 23(16), 6404-6412.

Saunders, N. R., Dziegielewska, K. M., Unsicker, K., \& Ek, C. J. (2016). Delayed astrocytic contact with cerebral blood vessels in FGF-2 deficient mice does not compromise permeability properties at the developing blood-brain barrier. Developmental Neurobiology, 76(11), 1201-1212. https://doi.org/10.1002/dneu.22383

Schroeter, M. L., Sacher, J., Steiner, J., Schoenknecht, P., \& Mueller, K. (2013). Serum S100B represents a new biomarker for mood disorders. Current Drug Targets, 14(11), 1237-1248.

Slattery, D. A., \& Cryan, J. F. (2012). Using the rat forced swim test to assess antidepressant-like activity in rodents. Nature Protocols, 7(6), 1009-1014. https://doi.org/10.1038/nprot.2012.044

Smyth, G. K. (2004). Linear models and empirical bayes methods for assessing differential expression in microarray experiments. Statistical Applications in Genetics and Molecular Biology, 3, Article3. https://doi. org/10.2202/1544-6115.1027

Sofroniew, M. V., \& Vinters, H. V. (2010). Astrocytes: Biology and pathology. Acta Neuropathologica, 119(1), 7-35. https://doi.org/10.1007/ s00401-009-0619-8

Srinivasan, B., Kolli, A. R., Esch, M. B., Abaci, H. E., Shuler, M. L., \& Hickman, J. J. (2015). TEER measurement techniques for in vitro barrier model systems. Journal of Laboratory Automation, 20(2), 107-126. https://doi.org/10.1177/2211068214561025

Stogsdill, J. A., Ramirez, J., Liu, D. I., Kim, Y. H., Baldwin, K. T., Enustun, E., ... Eroglu, C. (2017). Astrocytic neuroligins control astrocyte morphogenesis and synaptogenesis. Nature, 551(7679), 192-197. https:// doi.org/10.1038/nature24638

Strelau, J., Bottner, M., Lingor, P., Suter-Crazzolara, C., Galter, D., Jaszai, J., ... Unsicker, K. (2000). GDF-15/MIC-1 a novel member of the TGFbeta superfamily. Journal of Neural Transmission. Supplementum, (60), 273-276.

Strelau, J., Strzelczyk, A., Rusu, P., Bendner, G., Wiese, S., Diella, F., ... Unsicker, K. (2009). Progressive postnatal motoneuron loss in mice lacking GDF-15. Journal of Neuroscience, 29(43), 13640-13648. https://doi.org/10.1523/JNEUROSCI.1133-09.2009

Tanasic, S., Mattusch, C., Wagner, E. M., Eder, M., Rupprecht, R., Rammes, G., \& Di Benedetto, B. (2016). Desipramine targets astrocytes to attenuate synaptic plasticity via modulation of the ephrinA3/EphA4 signalling. Neuropharmacology, 105, 154-163. https:// doi.org/10.1016/j.neuropharm.2016.01.021

Wan, C., Liu, J., Nie, X., Zhao, J., Zhou, S., Duan, Z., ... Xu, G. (2014). 2, 3, 7, 8-Tetrachlorodibenzo-P-dioxin (TCDD) induces premature senescence in human and rodent neuronal cells via ROS-dependent mechanisms. PLoS ONE, 9(2), e89811. https://doi.org/10.1371/journ al.pone.0089811

Watkins, S., Robel, S., Kimbrough, I. F., Robert, S. M., Ellis-Davies, G., \& Sontheimer, H. (2014). Disruption of astrocyte-vascular coupling and the blood-brain barrier by invading glioma cells. Nature Communications, 5, 4196. https://doi.org/10.1038/ncomms5196

Wegener, G., Mathe, A. A., \& Neumann, I. D. (2012). Selectively bred rodents as models of depression and anxiety. Current Topics in Behavioral Neurosciences, 12, 139-187. https://doi.org/10.1007/7854_2011_192

Wu, G. Y., Deisseroth, K., \& Tsien, R. W. (2001). Spaced stimuli stabilize MAPK pathway activation and its effects on dendritic morphology. Nature Neuroscience, 4(2), 151-158. https://doi.org/10.1038/83976 


\section{SUPPORTING INFORMATION}

Additional Supporting Information may be found online in the Supporting Information section.

FIGURE S1 Test of the in situ hybridization probe generated to label and track gdf15 expression. Representative images of cortical brain slices derived from NAB rats depict the choroid plexus and BV labelled with DAPI to stain single nuclei (in blue, $i$ and iii) and with a probe against gdf15 mRNA (dashed lines; in magenta, ii and iv). It is evident the specificity of labelling in the choroid plexus, as well as the expression in filamentous domains around BV and cells around (which might represent astrocytes) (iv). Scale bar $100 \mu \mathrm{m}$ (i) and $50 \mu \mathrm{m}$ (iii)

FIGURE S2 Intensity of gdf15 does not change between NAB and HAB brains neither before nor after drug treatment. Using ISH we evaluated whether differences in gdf15 expression may occur between $N A B$ and $H A B$ brains before and after treatment with FLX. Our results showed that neither condition was affecting gdf15 expression. Data information: data are presented as mean $\pm S D$; one-way ANOVA, $p=0.2707$ followed by post hoc Fisher's LSD test. Table 1 for statistic details

FIGURE S3 Astrocyte numbers are lower in HAB vs. NAB brains, but FLX does not change their numbers after short-term treatment. Using IF-IHC we measured numbers of astrocytes $\left(\mathrm{GFAP}^{+}+\mathrm{S} 100 \mathrm{~B}^{+} /\right.$ $\mathrm{DAPI}^{+}$cells) in the PFC of NAB and HAB brains and revealed their reduced numbers, which were not reversed by a 48 hours FLX treatment. Data information: one-way ANOVA, $p=0.0134$ followed by post hoc Fisher's LSD test. Table 1 for statistic details Transparent Peer Review Report

Transparent Science Questionnaire for Authors

How to cite this article: Malik VA, Zajicek F, Mittmann LA, et al. GDF15 promotes simultaneous astrocyte remodeling and tight junction strengthening at the blood-brain barrier. J Neuro Res. 2020;98:1433-1456. https://doi.org/10.1002/ $\underline{\text { jnr.24611 }}$ 